Portland State University

PDXScholar

\title{
Multimodal Internal Waves Generated over a Subcritical Ridge: Impact of the Upper-Ocean Stratification
}

\author{
Xie Jieshuo \\ Chinese Academy of Sciences \\ Jiayi Pan \\ Chinese University of Hong Kong \\ David A. Jay \\ Portland State University, djay@pdx.edu
}

Follow this and additional works at: https://pdxscholar.library.pdx.edu/cengin_fac

Part of the Hydraulic Engineering Commons

Let us know how access to this document benefits you.

\section{Citation Details}

Jieshuo Xie, Jiayi Pan, and David A. Jay, 2015: Multimodal Internal Waves Generated over a Subcritical Ridge: Impact of the Upper-Ocean Stratification. J. Phys. Oceanogr., 45, 904-926.

This Article is brought to you for free and open access. It has been accepted for inclusion in Civil and Environmental Engineering Faculty Publications and Presentations by an authorized administrator of PDXScholar. Please contact us if we can make this document more accessible: pdxscholar@pdx.edu. 


\title{
Multimodal Internal Waves Generated over a Subcritical Ridge: Impact of the Upper-Ocean Stratification
}

\author{
JIESHUO XIE \\ State Key Laboratory of Tropical Oceanography, South China Sea Institute of Oceanology, Chinese Academy of \\ Sciences, Guangzhou, and Institute of Space and Earth Information Science, Chinese University of Hong Kong, \\ Hong Kong, and University of Chinese Academy of Sciences, Beijing, China \\ JIAYI PAN \\ Institute of Space and Earth Information Science, Chinese University of Hong Kong, Hong Kong, China \\ DAVID A. JAY \\ Department of Civil and Environmental Engineering, Portland State University, Portland, Oregon
}

(Manuscript received 30 June 2014, in final form 4 November 2014)

\begin{abstract}
Interaction of barotropic tides with subsurface topography is vital to ocean mixing. Yet the behavior of largeamplitude, nonlinear, internal solitary waves (ISWs) that can cause strong mixing remains poorly understood, especially that of higher-mode and multimodal internal waves. Therefore, a 2.5-dimensional, nonhydrostatic model with adjustable vertical resolution was developed to investigate effects of upper-ocean stratification on tidally induced multimodal internal waves and to show how they are generated by the subcritical ridge where only upward-propagating internal wave beams (IWBs) are present. The effects of the stratification on properties and characteristics of the excited IWBs and on the energy partition of the radiated mode- 1 and mode- 2 internal waves were investigated based on the model results. Higher modes of internal waves can also be effectively generated in the IWBs by the subcritical topography, and the contribution to IWBs from higher modes increases with the upper-ocean stratification. Mode-2 ISWs can be excited from the IWBs if both the tidal Froude number $\operatorname{Fr}_{2}^{0}=U_{0} / c_{2}$ and the contribution to IWBs from mode- 2 waves are sufficiently high $\left(U_{0}\right.$ is the tidal current speed, and $c_{2}$ is the phase speed of mode- 2 waves). In a moderately stratified upper ocean, both mode- 1 and mode- 2 ISWs can be produced, but for weak (strong) stratification, only mode-1 (mode-2) ISWs are generated. Further, it is found that the distance between two successive mode- 1 or mode-2 ISW trains increases linearly with the upper-ocean stratification. The ratio of the kinetic energy to the available potential energy for the mode-2 ISWs increases with the upper-ocean stratification in a strongly stratified ocean.
\end{abstract}

\section{Introduction}

\section{a. Scope}

Ocean stratification greatly influences the dynamical properties of internal tides, including their expressions in the surface elevation (Hall et al. 2013; Colosi and Munk 2006). In particular, the generation and propagation of internal wave beams (IWBs) and internal solitary waves (ISWs) are affected by stratification. But

Corresponding author address: Jiayi Pan, Institute of Space and Earth Information Science, Chinese University of Hong Kong, Shatin, N. T., Hong Kong, China.

E-mail:panj@cuhk.edu.hk the role of stratification on the generation and energy partition of IWBs and multimodal ISWs remains poorly understood. Here, we use a 2.5-dimensional (2.5D), nonhydrostatic model to analyze stratification effects on tidally induced multimodal ISWs and to show how multimodal internal waves can be generated by subcritical topography where only upward-propagating IWBs are present.

The issues analyzed in this paper are as follows: First, we clarify how upper-ocean stratification affects the components and propagation routes of tidally induced IWBs radiated from a subcritical topography. When these upward-propagating IWBs impinge on the pycnocline, ISWs may evolve from the IWBs, depending on the 
tidal Froude number (the ratio of tidal current speed to the phase speed of multimodal internal waves). Then we elucidate how the upper-ocean stratification affects characteristics and energy partition of multimodal internal waves. Finally, we determine internal wave sensitivity to other important background parameters, including tidal amplitude, topographic slope, and depth of the pycnocline.

\section{b. Background}

ISWs are common phenomena in the oceans. Numerous in situ and remote sensing observations provide evidence of mode-1 ISWs (Osborne and Burch 1980; Jackson 2004; Cai et al. 2012) because they can be easily captured by using oceanographic instruments and remote sensing images. Mode-2 ISWs are occasionally observed (Akylas and Grimshaw 1992; Bogucki et al. 2005; Yang et al. 2009; Shroyer et al. 2010). Shroyer et al. (2010) showed that mode-2 ISWs have important impacts on the coastal environment and vertical fluxes of heat and nutrients, although they carry much less baroclinic energy than mode-1 ISWs.

Several generation mechanisms for mode-2 ISWs have been identified. For example, Helfrich and Melville (1986), Vlasenko and Alpers (2005), and Liu et al. (2013) suggested that mode-2 ISWs can emerge after mode-1 ISWs pass over a continental slope or a ridge. Stastna and Peltier (2005) found that mode-2 ISWs could be generated by resonant interaction of a depth-independent velocity field with topography, for example, by the interaction of the barotropic tide with a ridge (Vlasenko and Alpers 2005; Xie et al. 2010; Ramp et al. 2012). Laboratory studies by Mercier et al. (2012) showed that mode-2 ISWs can also occur as harmonics (e.g., at twice the forcing frequency).

Another characteristic of mode-2 ISWs is that they are usually followed by an oscillatory wave tail that consists of a series of short, linear, mode- 1 waves (Farmer and Smith 1980; Akylas and Grimshaw 1992; Vlasenko et al. 2010). Conversely, some studies indicated that mode-2 ISWs can appear behind mode-1 internal waves (Vlasenko et al. 2005; Yang et al. 2009) or in isolation under certain stratification conditions (Vlasenko and Alpers 2005; Yang et al. 2009; Xie et al. 2010).

Three parameters are likely significant to the analysis of the tidally induced internal waves. The first, $\operatorname{Fr}_{n}^{0}$, is a tidal Froude number that describes the background forcing field relative to a mode- $n$ wave; it is defined (Vlasenko et al. 2005; Kang 2010) as the ratio of the barotropic tidal current speed $U_{0}$ to the phase velocity of the mode- $n$ internal waves $c_{n}$ :

$$
\operatorname{Fr}_{n}^{0}=U_{0} / c_{n}
$$

When $\operatorname{Fr}_{n}^{0} \ll 1$, linear theory is valid for analyzing the internal waves; $c_{n}$ is calculated under a hydrostatic assumption (below). At intermediate Froude numbers $\left(\operatorname{Fr}_{n}^{0} \sim 1\right)$, nonlinearity becomes significant and nonlinear internal wave bores, weak unsteady lee waves, and ISWs can be generated, depending on the slope of the topography. At high Froude numbers $\left(\operatorname{Fr}_{n}^{0}>1\right)$, in addition to bores and ISWs, strong unsteady lee waves may be formed.

A second Froude number, the wave Froude number $\mathrm{Fr}_{n}$, measures the nonlinearity of the generated internal waves (Staquet and Sommeria 2002):

$$
\mathrm{Fr}_{n}=u_{n} / c_{n}
$$

where $u_{n}$ is the maximum horizontal current velocity associated with the mode- $n$ internal waves. In this paper, $\operatorname{Fr}_{n}^{0}$ and $\mathrm{Fr}_{n}$ are used, respectively, to describe the generation and nonlinear propagation of multimodal internal waves.

The final nondimensional parameter is the relative steepness of the topography $\gamma$, defined as (Buijsman et al. 2010; Kang 2010)

$$
\gamma=\max \left(\frac{h_{x}}{\alpha}\right)
$$

where $h_{x}$ is the topographic slope; $\alpha$ is the IWB slope, given by

$$
\alpha=\sqrt{\left(\sigma^{2}-f^{2}\right) /\left[N^{2}(z)-\sigma^{2}\right]},
$$

where $\sigma$ is the wave frequency, $f$ is the Coriolis parameter, and $N(z)$ is the buoyancy frequency. For $\gamma>1$, IWBs generated at a supercritical ridge will be directed both upward and downward (Lamb 2004; Nash et al. 2006). If the topography is subcritical $(\gamma<1)$, only upward-propagating IWBs can be generated (Balmforth et al. 2002). For topography with realistic stratification, New and Pingree (1990) found that IWBs that radiated upward and downward from topography could evolve into mode-1 ISWs when they impinged the thermocline. Similarly, Gerkema (2001) and Akylas et al. (2007) concluded that the generation of mode-1 ISWs was due to the scattering and reflecting of IWBs in the pycnocline. Recently, Grisouard et al. (2011) used a fully nonlinear, nonhydrostatic model in an idealized configuration to show how mode-1, mode-2, and mode-3 ISWs could be excited from previously generated IWBs.

\section{c. Structure}

The structure of the remainder of the paper is as follows: Section 2 describes the 2.5D model and its parameters. Section 3 analyzes how upper-ocean stratification affects the tidally induced multimodal internal waves. 
Sensitivity studies and result discussions are presented in sections 4 and 5, respectively. The results are summarized in section 6 .

\section{Model description}

a. Governing equations for internal waves and choice of the basic parameters

Considering a $2.5 \mathrm{D}(x, z)$ flow in a continuously stratified ocean, internal waves can be described by four governing Eqs. (5)-(8) (Vlasenko et al. 2005),

$$
\begin{gathered}
\xi_{t}+J(\xi, \psi)-f v_{z}=g \tilde{\rho}_{x} / \bar{\rho}_{0}+A^{H} \xi_{x x}+\left(A^{V} \xi_{z}\right)_{z}, \\
v_{t}+J(v, \psi)+f \psi_{z}=A^{H} v_{x x}+\left(A^{V} v_{z}\right)_{z}, \\
\tilde{\rho}_{t}+J(\tilde{\rho}, \psi)+\bar{\rho}_{0} / g N^{2}(z) \psi_{x} \\
=K^{H} \tilde{\rho}_{x x}+\left(K^{V} \tilde{\rho}_{z}\right)_{z}+\left(K^{V} \rho_{0 z}\right)_{z}, \quad \text { and } \\
\xi=\psi_{x x}+\psi_{z z},
\end{gathered}
$$

with five boundary and initial conditions:

$\psi=0, \quad \xi=0, \quad v_{n}=0, \quad \tilde{\rho}_{n}=0, \quad(z=0)$,

$\psi=\psi_{0} \sin (\sigma t), \quad \xi=0, \quad v_{n}=0, \quad \tilde{\rho}_{n}=0, \quad[z=H(x)]$,

$\psi=-z \psi_{0} \sin (\sigma t) / H_{0}, \quad \xi=0$,

$v=-\left[(f / \sigma) \psi_{0} \cos (\sigma t)\right] / H_{0}, \quad \tilde{\rho}=0, \quad(x=-L)$,

$\psi=-z \psi_{0} \sin (\sigma t) / H_{0}, \quad \xi=0$,

$v=-\left[(f / \sigma) \psi_{0} \cos (\sigma t)\right] / H_{0}, \quad \tilde{\rho}=0, \quad(x=L), \quad$ and

$\psi=0, \quad \xi=0, \quad v=-\left[(f / \sigma) \psi_{0}\right] / H(x), \quad \tilde{\rho}=0, \quad(t=0)$,

where $\xi$ is the vorticity, $\psi$ is the streamfunction, $(u, v, w)$ is the velocity vector, and the Coriolis parameter $f=$ $5.07 \times 10^{-5} \mathrm{~s}^{-1}$. Also, $A^{V}=10^{-6} \mathrm{~m}^{2} \mathrm{~s}^{-1}$ and $A^{H}=$ $10^{-5} \mathrm{~m}^{2} \mathrm{~s}^{-1}\left(K^{V}=10^{-6} \mathrm{~m}^{2} \mathrm{~s}^{-1}\right.$ and $\left.K^{H}=10^{-5} \mathrm{~m}^{2} \mathrm{~s}^{-1}\right)$ are the vertical and horizontal eddy viscosities (diffusivities), respectively; $J$ is the Jacobian operator; and $g$ is the acceleration due to gravity. Here, the sea surface is at $z=0, \sigma=2 \pi / T$ is the $\mathrm{M}_{2}$ tidal frequency $(T=12.42 \mathrm{~h})$, and $\psi_{0}$ is the vertically integrated volume flux for the $\mathrm{M}_{2}$ barotropic flow. A ridgelike bottom topography is defined by $H(x)=-\left[H_{0}-h_{0} \exp \left(-x^{2} / 2 W_{0}^{2}\right)\right]$, where $H_{0}=$ $200 \mathrm{~m}$ is the water depth away from the ridge, $W_{0}$ is the width of the ridge, and $h_{0}=60 \mathrm{~m}$ is the height of the ridge, located at a depth where the stratification does not vary significantly (Fig. 1). In this study, $\psi_{0}$ is set as $25 \mathrm{~m}^{2} \mathrm{~s}^{-1}$, and $W_{0}$ is $2 \mathrm{~km}$ unless otherwise stated. The term $L$ is the distance between the model domain boundary and the ridge center, and $L$ is sufficiently large so that the internal wave generation is unaffected by the boundary. Finally, $\rho_{0}(z)$ is the static density, $\bar{\rho}_{0}$ is the constant average of density, and $\tilde{\rho}$ is the density disturbance because of wave motion. The seawater density is written as $\rho(z)=\rho_{0}(z)+$ $\tilde{\rho}(z)$. Note that the calculation is $2.5 \mathrm{D}$ in the sense that $\partial(\cdot) / \partial y=0$, and $v$ is present because of the Coriolis force.

The governing Eqs. (5)-(13) are solved after making the following coordinate transform:

$$
x_{2}=x, \quad z_{2}=\int_{z}^{0} N(s) d s / \int_{H(x)}^{0} N(s) d s,
$$

where $s$ is a dummy vertical integration variable. The transformed equations are solved by using the alternative direction implicit (ADI) method at every temporal semistep. At the first (second) semistep, the differential scheme in the $x$ direction is made implicit (explicit), while that in the $y$ direction is kept explicit (implicit); the details of the ADI method can be found in Kantha and Clayson (2000). In this study, the model run is implemented with a horizontal spatial resolution $\Delta x=$ $25 \mathrm{~m}$ to satisfy the horizontal resolution criterion for nonhydrostatic internal wave modeling (Vitousek and Fringer 2011) and with the time step $\Delta t=5 \mathrm{~s}$ to satisfy the Courant-Friedrichs-Lewy (CFL) condition. The resulting Courant number satisfies $C_{r}=\left(c_{n} \Delta t\right) / \Delta x \leq 0.28$ for all of the stratification cases based on the upper limit of the internal wave speeds (below) of all the stratification cases. The total grid number varies from 8000 to 17200 in different simulation cases, depending on model domain. The number of vertical levels in the computational domain is 50 .

\section{b. Density and buoyancy frequency profiles}

The upper ocean is generally more stratified than deeper waters (e.g., Levitus 2009). Thus, it is reasonable to assume that the strong stratification appears mainly above the ridge crest with which the internal waves interact, as shown in Fig. 1. In Fig. 1, $D$ represents the distance between the pycnocline and ridge crest and is set as $75 \mathrm{~m}$. Thus, the pycnocline depth $h$ is $-65 \mathrm{~m}$. The stratification is designed based on Xie et al. (2010):

$$
N^{2}(z)=\frac{g \Delta \rho_{0}}{\delta \rho_{h}} \operatorname{sech}^{2}\left[\frac{2(z-h)}{\delta}\right],
$$

where $\delta$ is the pycnocline width; $\Delta \rho_{0}$ is the density difference; and $\rho_{h}$ is the density value at the pycnocline depth $h$. The stratification is adjusted with the varying parameters listed in Table 1, which makes 15 stratification cases $\mathrm{N} i$ ( $i=1$ to 15 ) shown in Fig. 2, representing 


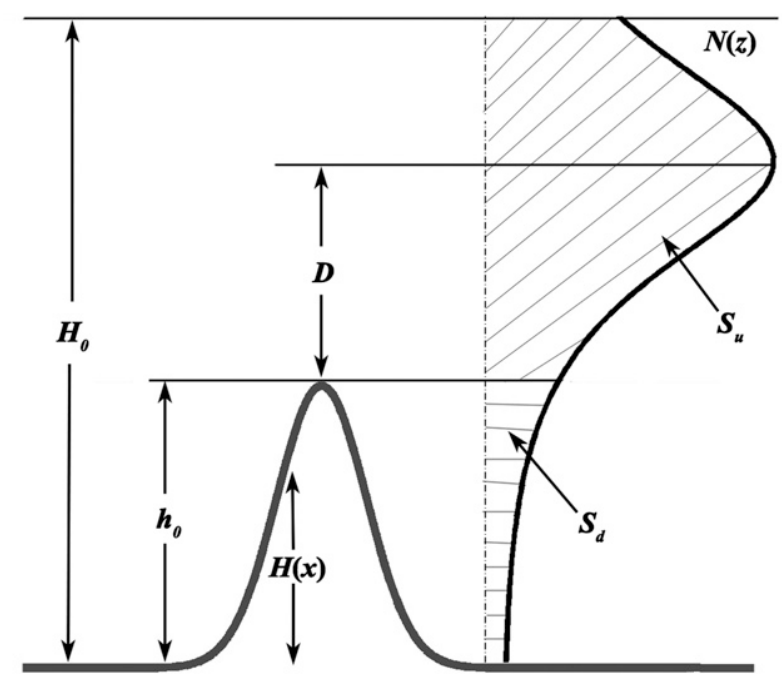

FIG. 1. Sketch of the buoyancy frequency $N(z)$ profile and the computational domain with a Gaussian ridge used in this study.

a wide range of the upper-ocean stratification. A slight adjustment is performed to ensure that all the stratifications are unchanged below the ridge top. In this study, a nondimensional parameter $R$ is introduced to simplify the quantification of stratification. It is defined as $R=$ $\bar{N}_{u} / \bar{N}_{d}$ and listed in Table 1, where $\bar{N}_{u}=S_{u} /\left(H_{0}-h_{0}\right)$ $\left(\bar{N}_{d}=S_{d} / h_{0}\right)$ represents the averaged stratification above (below) the ridge with $S_{u}=\int_{h_{0}-H_{0}}^{0} N(s) d s\left[S_{d}=\right.$ $\left.\int_{-H_{0}}^{h_{0}-H_{0}} N(s) d s\right]$, denoting the vertical integral of the stratification above (below) the ridge (Fig. 1). In Fig. 2, $S_{u}$ changes gradually from 0.56 to $2.56 \mathrm{~m} \mathrm{~s}^{-1}$, as listed in Table 1. Unlike $S_{u}$ above the ridge crest, $S_{d}$ is held constant at $0.076 \mathrm{~m} \mathrm{~s}^{-1}$. The 15 stratification profiles used in this study are referred to as cases $\mathrm{N} 1$ to $\mathrm{N} 15 ; N_{u}$ and $R$ increase continuously from $\mathrm{N} 1$ to $\mathrm{N} 15$.

\section{c. Linear wave phase speed $c_{n}$ and tidal Froude number $\operatorname{Fr}_{n}^{0}$}

The linear long-wave phase speed $c_{n}$ for mode- $n$ internal waves is estimated by solving the eigenvalue equation under the hydrostatic approximation:

$$
\begin{aligned}
& \varphi_{n}^{\prime \prime}(z)+\frac{N^{2}(z)}{c^{2}} \varphi_{n}(z)=0 \\
& \varphi_{n}(0)=0, \quad \varphi_{n}\left(-H_{0}\right)=0 .
\end{aligned}
$$

For the initial (undisturbed) buoyancy frequency profiles in Fig. 2, the linear long-wave speeds of the two leading modes $c_{n}(n=1,2)$ are shown in Fig. 3a; the corresponding tidal Froude numbers $\mathrm{Fr}_{n}^{0}=U_{c} / c_{n}$ for maximum tidal flow $U_{c}$ at the ridge crest are displayed in Fig. 3b. The peak tidal flow is subcritical (supercritical) for linear mode- $n$ waves if $\operatorname{Fr}_{n}^{0}<1\left(\operatorname{Fr}_{n}^{0}>1\right)$.

\section{Model results and analysis}

\section{a. Tidally induced IWBs}

Simulated density and horizontal velocity fields are displayed for representative cases $\mathrm{N} 2, \mathrm{~N} 5$, and $\mathrm{N} 10$ at $t=$ $3 T$ (Fig. 4); characteristic lines computed using Eq. (4) are also shown. (Since the characteristics of internal waves excited on both sides of the ridge are almost the same, only model results on the right side of the ridge are analyzed below.) The simulated IWBs, a superposition of multimodal internal waves (Gerkema and Zimmerman 2008), are consistent with the characteristic lines estimated from linear theory. At time $t=3 T$, the two beams emerge just to the right of the ridge crest, which is different from some observations; for example, Pickering and Alford (2012) observed that IWBs emerged directly above the crest of Kaena Ridge. This shift in beam origin is because of a Doppler shift associated with the oscillatory tidal current; this can be demonstrated by the results for case N11 (Fig. 5). Simulated density, horizontal velocity fields, and IWBs are shown in Fig. 5 for a tidal period from 2.5T to 3.5T with an interval of $0.25 T$. Figure 5 reveals that the two IWBs emerge directly above the ridge crest at $t=2.75 T$ and $t=$ 3.25T. Because of the Doppler shift from the rightward (leftward) transport of the barotropic tide, the IWBs shift to the right (left) of the ridge crest at $t=3 T(t=3.5 T)$. However, the Doppler shift does not alter the slopes of the characteristic lines, which depend primarily on the stratification $N_{u}$. Only upward-propagating beams are generated in all the numerical results. This occurs because the relative steepness of the topography Eq. (3) for all cases is $\gamma=0.222<1$, so the topography is subcritical.

Figure 4 shows that the character of the tidally excited IWBs varies with stratification. Variations in beam

\begin{tabular}{|c|c|c|c|c|c|c|c|c|c|c|c|c|c|c|c|}
\hline Cases & N1 & $\mathrm{N} 2$ & N3 & N4 & N5 & N6 & N7 & N8 & N9 & N10 & N11 & N12 & N13 & N14 & N15 \\
\hline$\delta(\mathrm{m})$ & 90 & 82 & 78 & 74 & 71.5 & 69 & 67 & 64.8 & 63.3 & 62 & 60.9 & 60 & 58 & 54 & 52 \\
\hline$\Delta \rho_{o}\left(\mathrm{~kg} \mathrm{~m}^{-3}\right)$ & 0.25 & 0.40 & 0.51 & 0.66 & 0.81 & 1.00 & 1.25 & 1.48 & 1.73 & 2.00 & 2.25 & 2.50 & 3.20 & 5.00 & 6.50 \\
\hline$\rho_{h}-1000\left(\mathrm{~kg} \mathrm{~m}^{-3}\right)$ & 25.26 & 25.20 & 25.14 & 25.07 & 25.00 & 24.91 & 24.79 & 24.68 & 24.56 & 24.43 & 24.31 & 24.19 & 23.85 & 22.97 & 22.24 \\
\hline$S_{u}\left(\mathrm{~m} \mathrm{~s}^{-1}\right)$ & 0.55 & 0.69 & 0.77 & 0.87 & 0.96 & 1.06 & 1.18 & 1.28 & 1.38 & 1.47 & 1.56 & 1.64 & 1.84 & 2.26 & 2.56 \\
\hline$R$ & 3.1 & 3.9 & 4.4 & 4.9 & 5.4 & 6.0 & 6.7 & 7.2 & 7.8 & 8.3 & 8.8 & 9.3 & 10.4 & 12.8 & 14.4 \\
\hline
\end{tabular}

TABLE 1. Parameters $S_{u}$ and $R$ for stratification cases N1 to N15. 

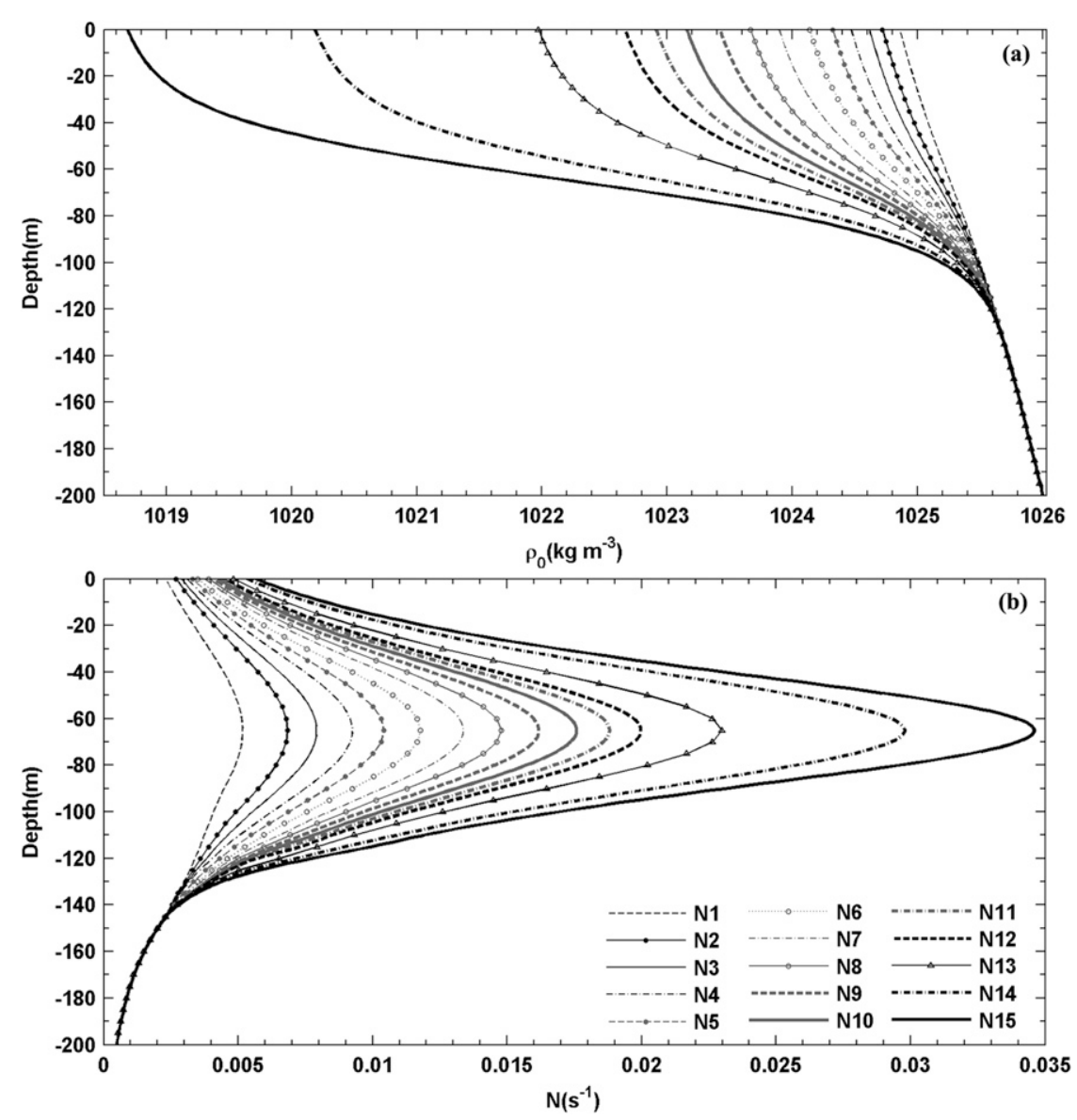

FIG. 2. The 15 (a) density and (b) stratification profiles N1 to N15 for this study.

content are investigated using the empirical orthogonal functions (EOFs); the EOF method is conventionally used in oceanographic studies to analyze spatialtemporal characteristics of a physical parameter (North et al. 1982). The method is also used in analyzing internal waves when the normal-mode approach fails (Vazquez et al. 2006; Venayagamoorthy and Fringer 2007). For each case, we apply the EOF analysis to the vertical velocity in a water column at the horizontal location where the first upward-crossing IWB originating from the ridge reaches the pycnocline depth $(z=$ $-65 \mathrm{~m})$, namely, at the horizontal location of the intersection of the first upward-crossing characteristic line with the depth of $z=-65 \mathrm{~m}$ for the time period of $t=$ $2.5 T$ to $4.5 T$. In this study, it is found that ISWs are not present in this water column; they only appear after the first upward crossing IWBs are scattered by the pycnocline, as shown in Figs. 4 and 5, so that the EOF analysis results reveal only the characteristics of the linear, multimodal internal waves in the IWBs.

The normalized spatial EOF modes of vertical velocity for representative cases $\mathrm{N} 2, \mathrm{~N} 5$, and N10 are shown in Fig. 6; the EOF spatial structure is qualitatively consistent with the structure of the normal modes. The percentages of the total variance accounted for by the leading six normal modes, determined by the number of zero crossings minus one for $R \leq \sim 10.0$ (i.e., cases $\mathrm{N} 1$ to N12), are shown in Fig. 7. However, the EOF structures for higher stratification cases with $R>\sim 10.0$ (i.e., cases $\mathrm{N} 13$ to N15) differ from that of normal modes (figure not shown here). For the stratifications with $R>\sim 10.0$, the first EOF mode corresponds to the second normal mode, while the second EOF mode corresponds to the first normal mode. The EOF analysis shows that for these stronger stratification cases, the contribution of the first internal mode to the IWBs decreases, while those of the higher internal modes (e.g., mode 2) increase and exceed that of the first internal mode. The variation of multimodal contents derived by the EOF analysis and the tidal Froude number $\operatorname{Fr}_{n}^{0}$ are useful in understanding the generation of the multimodal internal waves (below).

The stratification below the ridge top is the same in all cases analyzed here. Still, the variation of multimode 

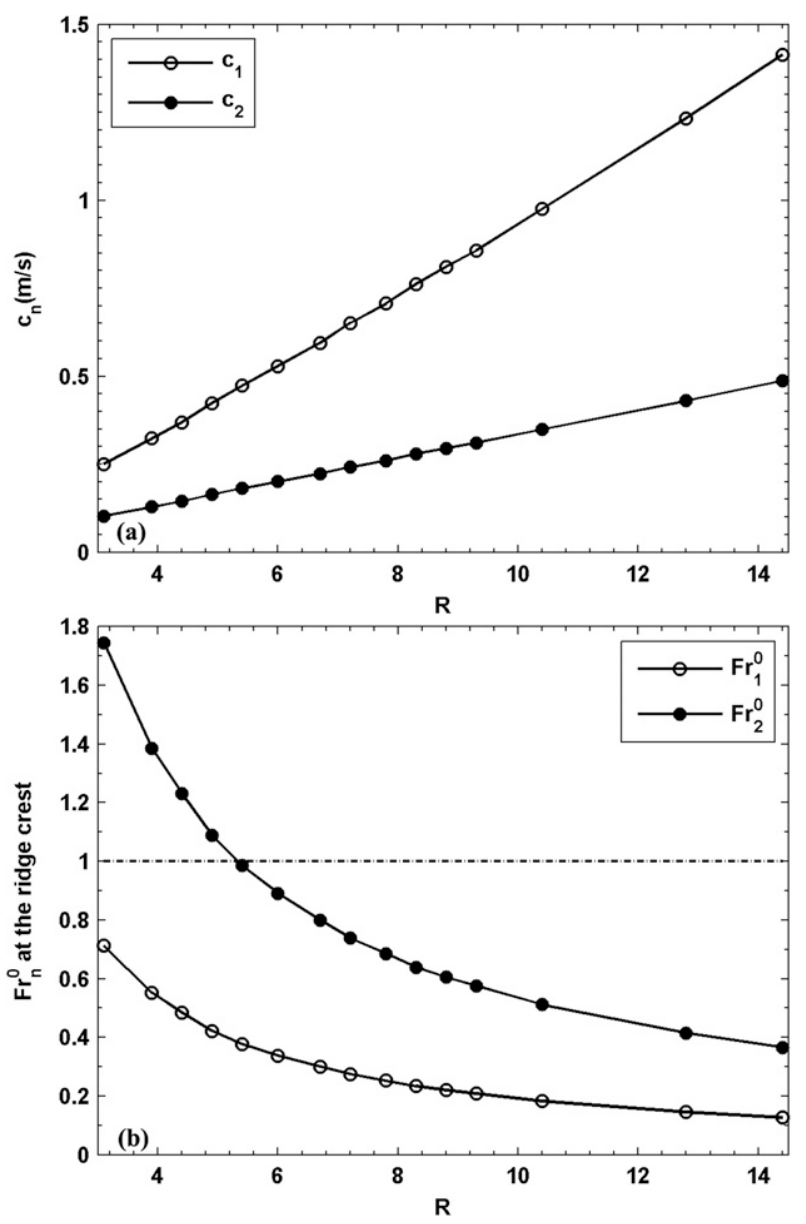

FIG. 3. (a) Hydrostatic linear long-wave speeds and (b) tidal Froude numbers of the first two modes of internal waves for all the stratification profiles N1 to N15.

contents in the IWBs with $R$ shown by the EOF analysis reveals that the generation of the IWBs can be affected by the upper-ocean stratification. This effect can be further explored by using the barotropic-to-baroclinic energy conversion $C$. The term $C$ is proportional to the product of the barotropic vertical velocity and the baroclinic perturbation pressure evaluated at the bottom boundary (Nash et al. 2006; Buijsman et al. 2012). The tidally averaged value of $C$ integrated over the domain enclosing the ridge can be written as

$$
C=\int\left\langle p^{\prime}(z=H) W\right\rangle d x,
$$

where $p^{\prime}(z=H)$ is the perturbation pressure at the bottom $z=H(x), W=(\partial H / \partial x) U$ is the barotropically induced depth-integrated vertical velocity, $U$ is the depth mean of $u$, and $\langle\cdots\rangle$ represents the temporal average over a tidal cycle. The energy conversion $C$ versus $R$ is shown in Fig. 8 for all cases N1 to N15. The barotropic-to-baroclinic energy conversion increases with upper-ocean stratification because the change in the upper-ocean stratification impacts the perturbation pressure $p^{\prime}$ at the bottom. This result implies that the baroclinic energy of the IWBs strengthens as the upper-ocean stratification becomes stronger; therefore, the radiated multimodal internal waves can potentially be affected by the upper-ocean stratification although the stratification is held constant below the ridge top.

\section{b. Characteristics of radiated internal waves}

Previous studies have showed that mode-1 ISWs are generated by scattering of IWBs in the pycnocline (e.g., Gerkema 2001; Akylas et al. 2007). We have shown (in Figs. 4, 5) that mode-2 ISWs may also be scattered and radiated out from tidally excited IWBs, and both the radiated mode- 1 and mode- 2 ISWs appear mainly after the first upward crossing of the IWBs. We explore, therefore, stratification effects on characteristics and energy partition of the radiated mode- 1 and mode- 2 internal waves that are excited during the initial tidal cycles and are stable at $t=3 T$ (Fig. 4). Note that the first two modes of internal waves excited during the initial tidal cycles have been scattered and radiated out from the IWBs at this moment.

The distances between two successive wave trains for the first- and second-mode waves (labeled as $L_{1}$ and $L_{2}$ in Fig. 4b) that reflect the internal wave propagation characteristics can be used as a characteristic horizontal length for internal wave generation and are affected by the stratification. Based on the linear theory (e.g., Llewellyn Smith and Young 2002), the distances between the two successive wave trains can be expressed approximately as $L_{n}=\left(\bar{N} H_{0} / n \pi\right) T$, where $\bar{N}=$ $1 / H_{0} \int_{-H_{0}}^{0} N(s) d s$. Thus, they are related to variations in phase speed with changing stratification, and the ratio $L_{2} / L_{1}$ should be $1 / 2$ for arbitrary stratification. In this study, these simulated distances are plotted versus $R$ in Fig. 9a. Here, the optimum linear fit equations for $L_{1}$ and $L_{2}$ versus $R$ are $L_{1}=1.96 R-3.85$ and $L_{2}=$ $0.60 R+1.50$, respectively. Thus, $L_{1}$ and $L_{2}$ both increase linearly with the stratification above the ridge. Also, the ratio $L_{2} / L_{1}$ is approximately inversely proportional to the stratification above the ridge (Fig. 9b). Note that in this study, $0.34<L_{2} / L_{1}<0.46$, close to the linear theory result of $L_{2} / L_{1} \sim 1 / 2$.

The distance $L_{1}$ is used as a length scale for a nondimensional coordinate system that may better specify the internal wave dynamical regimes, as shown in Fig. 10. Figure 10 reveals that the effect of stratification on the internal waves can be divided into three regimes based on $R$ : 


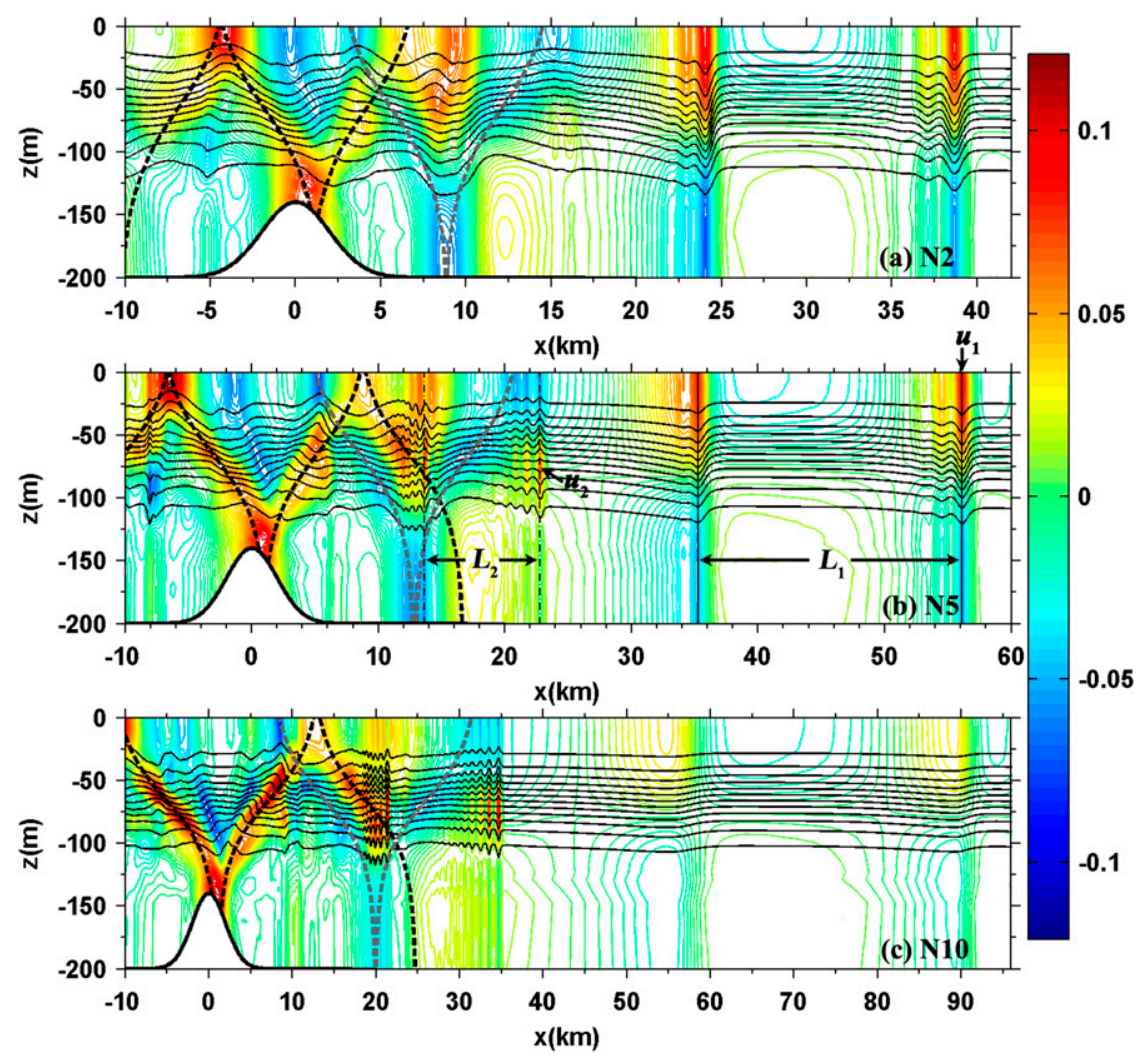

FIG. 4. Simulated density and horizontal velocity fields corresponding to cases (a) N2, (b) N5, and (c) N10 at $t=3 T$. The gray and black dashed curves are the characteristic lines computed by Eq. (4). (Note the difference in the horizontal scales due to the different horizontal wavelengths for different upper-ocean stratifications.)

Regime I, where $R \leq \sim 4.0$ (cases 1 to 2 ). In this regime, the tidal Froude number for the mode- 1 internal waves is close to 1 (i.e., $\mathrm{Fr}_{1}^{0} \sim 1$; Fig. 3b), so mode-1 ISWs can be generated. However, although the tidal Froude number for mode- 2 internal waves is $>1$ (i.e., $\mathrm{Fr}_{2}^{0}>1$ ), only a mode- 2 baroclinic tide is generated and no mode-2 ISW appears (Fig. 10b). While this may seem inconsistent with the tidal Froude number criterion, it occurs because the percentage contribution (Fig. 7) to the IWBs from the mode- 2 component is too low to allow the nonlinear evolution of the mode-2 ISWs.

Regime II, where $\sim 4.0 \leq R \leq \sim 7.5$ (cases 3 to 8 ). In this regime, both tidal Froude numbers are $O(1)$ $\left(\mathrm{Fr}_{1}^{0} \sim 1\right.$ and $\mathrm{Fr}_{2}^{0} \sim 1$; Fig. $\left.3 \mathrm{~b}\right)$, and the percentage contributions from the two internal wave modes are favorable to the formation of mode- 1 and mode- 2 ISWs (Figs. 10c-e). However, the mode-1 ISWs are weaker than those in regime I. Mode-1 ISWs gradually weaken with increasing $R$ because $\mathrm{Fr}_{1}^{0}$ (Fig. 3b) and the percentage contribution to total internal wave variance from the mode- 1 component (Fig. 7) both decrease. As $R$ increases, mode-2 ISWs become more prominent, as implied by the increase of percentage contribution from the mode- 2 waves (Fig. 7).

Regime III, where $R \geq \sim 7.5$ (cases 9 to 15). In this regime, the tidal Froude number for mode- 1 internal waves is too small $\left(\mathrm{Fr}_{1}^{0} \ll 1\right)$ to support the evolution of the mode-1 ISWs, although the variance contribution from the mode- 1 component is still substantial. This leads to generation of a mode1 baroclinic tide but not mode-1 ISWs. Mode-2 ISWs still appear in this regime because of the nearly critical tidal Froude number and the high percentage contribution from the mode- 2 waves (Fig. 7), but the strength of the mode-2 ISWs is reduced as $\mathrm{Fr}_{2}^{0}$ decreases (Fig. 3b), similar to that of the situation for mode-1 ISWs in regime II.

\section{c. Maximum current velocity $u_{n}$ and wave Froude number $\mathrm{Fr}_{n}$}

Internal wave velocities are important for transport and mixing processes, but they vary in a complicated 

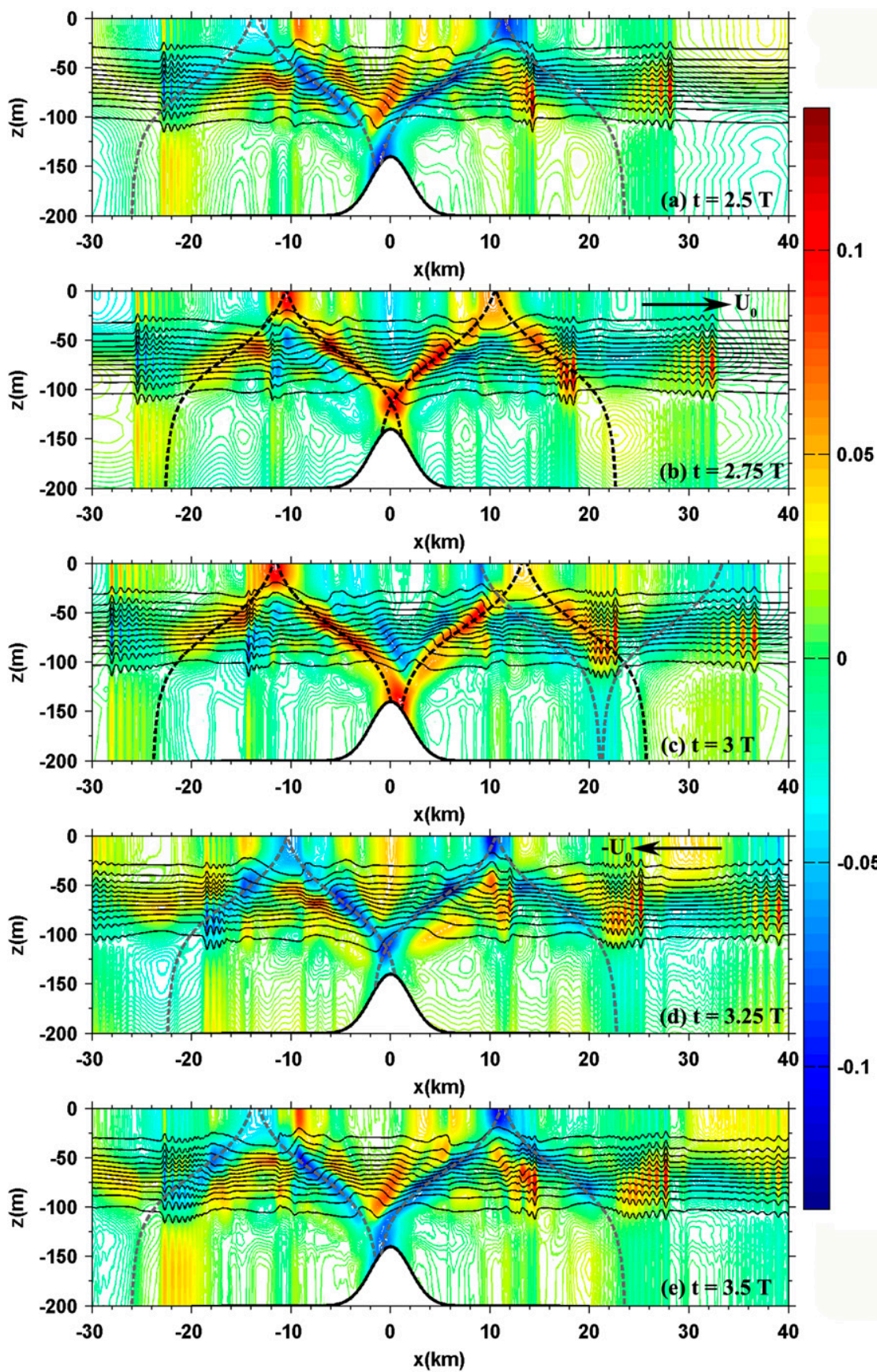

FIG. 5. Simulated density and horizontal velocity fields for stratification N11; IWBs over a tidal period (a)-(e) from $t=2.5 T$ to $t=3.5 T$ at intervals of $0.25 T$ are shown. The gray and black dashed curves are the characteristic lines computed from linear theory Eq. (4). The emergence point of the beams shifts with the barotropic tidal currents. Arrows show direction and strength of the barotropic current. The strength of the barotropic current reaches maximum at $t=2.75 T$ and $t=3.25 T$ and is zero at $t=2.5 T, 3 T$, and 3.5T.

manner with mode number and $R$. The maximum horizontal current velocity $u_{n}(n=1,2)$ of the mode- $n$ waves after they are scattered and radiated out from the tidally excited IWBs are shown in Fig. 11a. Here, $u_{1}$ appears at the top of the water column, while $u_{2}$ appears below the pycnocline; typical examples of the depths of the $u_{n}$ are shown in Fig. 4b. The wave Froude number $\mathrm{Fr}_{n}$ from Eq. (2) for the two leading modes of ISWs is then computed 


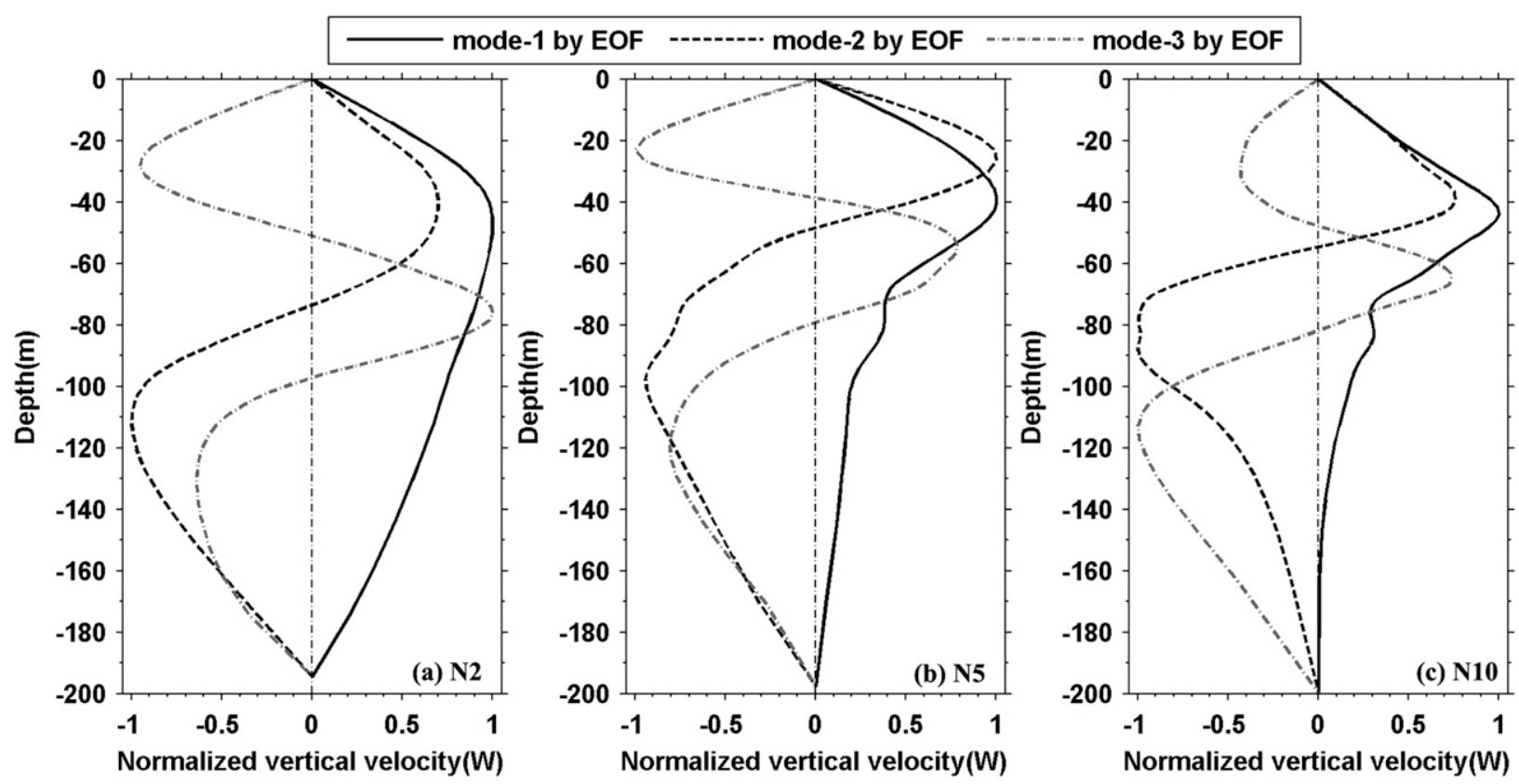

FIG. 6. The normalized structures of vertical velocity derived by the EOF method for cases (a) N2, (b) N5, and (c) N10 for modes 1 to 3.

(Figs. 11b). As indicated by $\mathrm{Fr}_{1}$, the nonlinearity of the mode-1 internal wave decreases with $R$ from a maximum of $\mathrm{Fr}_{1}=0.325$ at $R=3.1$, consistent with the density field disturbance of the mode- 1 waves in Fig. 10. For the mode-2 internal waves, the wave nonlinearity indicated by $\mathrm{Fr}_{2}$ increases with $R$ up to $\mathrm{Fr}_{2}=0.46$ at $R=\sim 7.2$. Then, it decreases when $\mathrm{R} \geq \sim 7.8$; however, the maximum isopycnal displacement caused by the mode2 ISWs decreases less than the $\mathrm{Fr}_{2}$ (e.g., in Figs. 10f-h; i.e., for $\sim 7.8 \leq R \leq \sim 10.4$ ). This occurs because the wave Froude number reflects primarily the nonlinearity due to the velocity field, and the slight decrease of the maximum isopycnal displacement for $\sim 7.8 \leq R \leq \sim 10.4$ actually reflects the variation of the unequal partition between

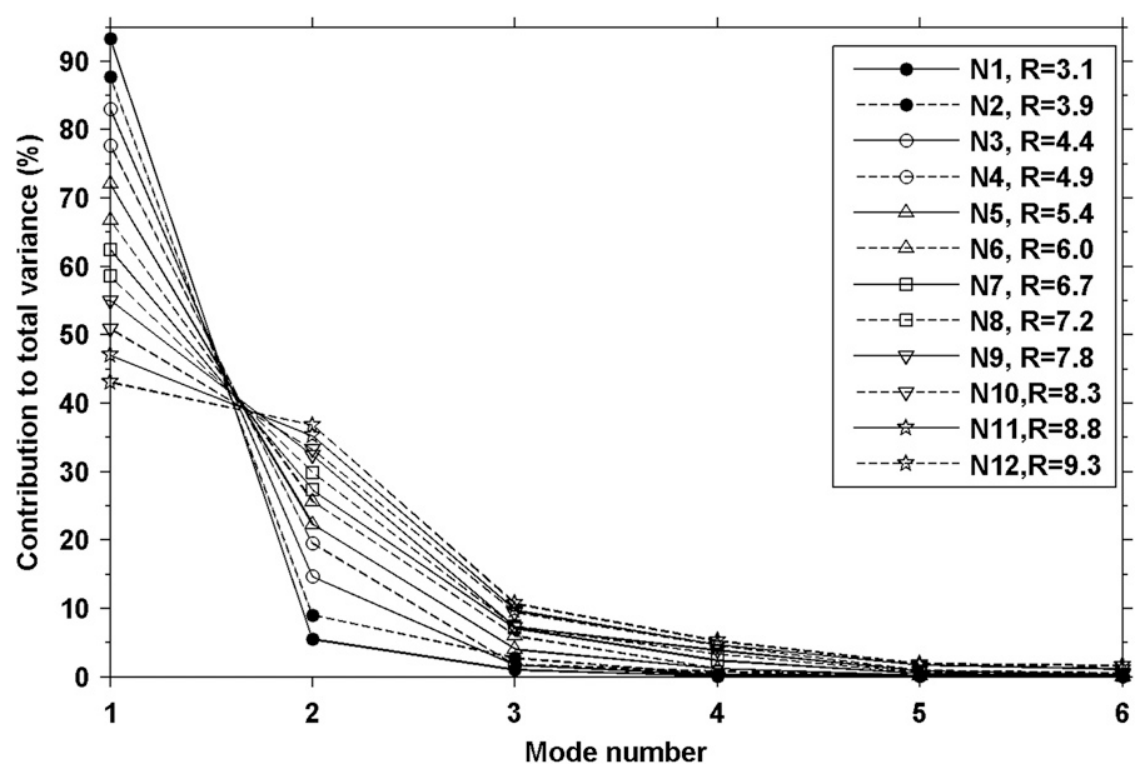

FIG. 7. The percentage contributions of the leading six modes to the total variance for cases $\mathrm{N} 1$ to $\mathrm{N} 12(R \leq \sim 10.0)$. (Note that the mode number is determined by the number of zero crossings minus one.) 


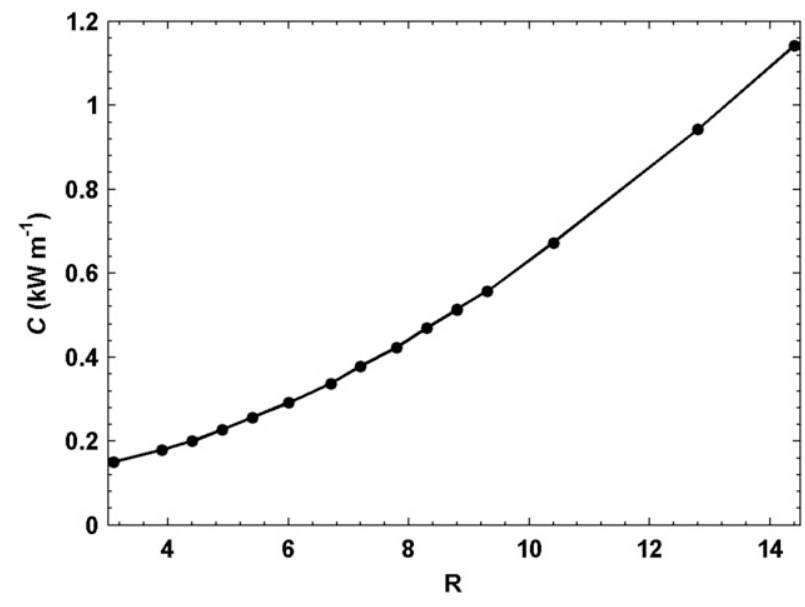

FIG. 8. The barotropic-to-baroclinic energy conversion $C$ vs the upper-ocean stratification parameter $R$.

wave kinetic and available potential energies, as investigated in section $3 \mathrm{~d}$.

\section{d. Energy relation in the radiated internal waves}

A linear wave exhibits an equipartition between kinetic and potential energy as an asymptotic condition. Departures from equipartition have implications for the wave nonlinearity. The baroclinic kinetic and available potential energies of the mode- $n(n=1,2)$ internal waves after they are scattered and radiated out from the tidally excited IWBs are computed over a scale length $L_{n}$ and are given by

$$
\mathrm{KE}_{n}=\int_{x_{n}}^{x_{n}+L_{n}} \int_{H(x)}^{0} E_{k} d z d x,
$$

and

$$
\mathrm{APE}_{n}=\int_{x_{n}}^{x_{n}+L_{n}} \int_{H(x)}^{0} E_{a} d z d x,
$$

where $E_{k}$ is the kinetic energy (KE) density, and $E_{a}$ is the available potential energy (APE) density defined as

$$
E_{a}(x, z, t)=g \int_{z}^{z *(x, z, t)}[\bar{\rho}(s)-\rho(x, z, t)] d s
$$

where $z^{*}(x, z, t)$ is the height of the water parcel at $(x, z)$ at time $t$ in the reference state, and $\bar{\rho}$ is the initial undisturbed density (Lamb 2008). The $L_{n}$ ( $n=1$ and 2 for mode- 1 and mode- 2 waves, respectively) specify the horizontal scale of the radiated linear or nonlinear internal modes. For this study, $x_{1}=2 L_{1}, x_{2}=0.8 L_{1}$, and the $L_{n}(n=1,2)$ versus $R$ are shown in Fig. 9a.

The calculated baroclinic energies for all 15 cases are shown in Fig. 12a, in which the baroclinic energies $E_{1}$
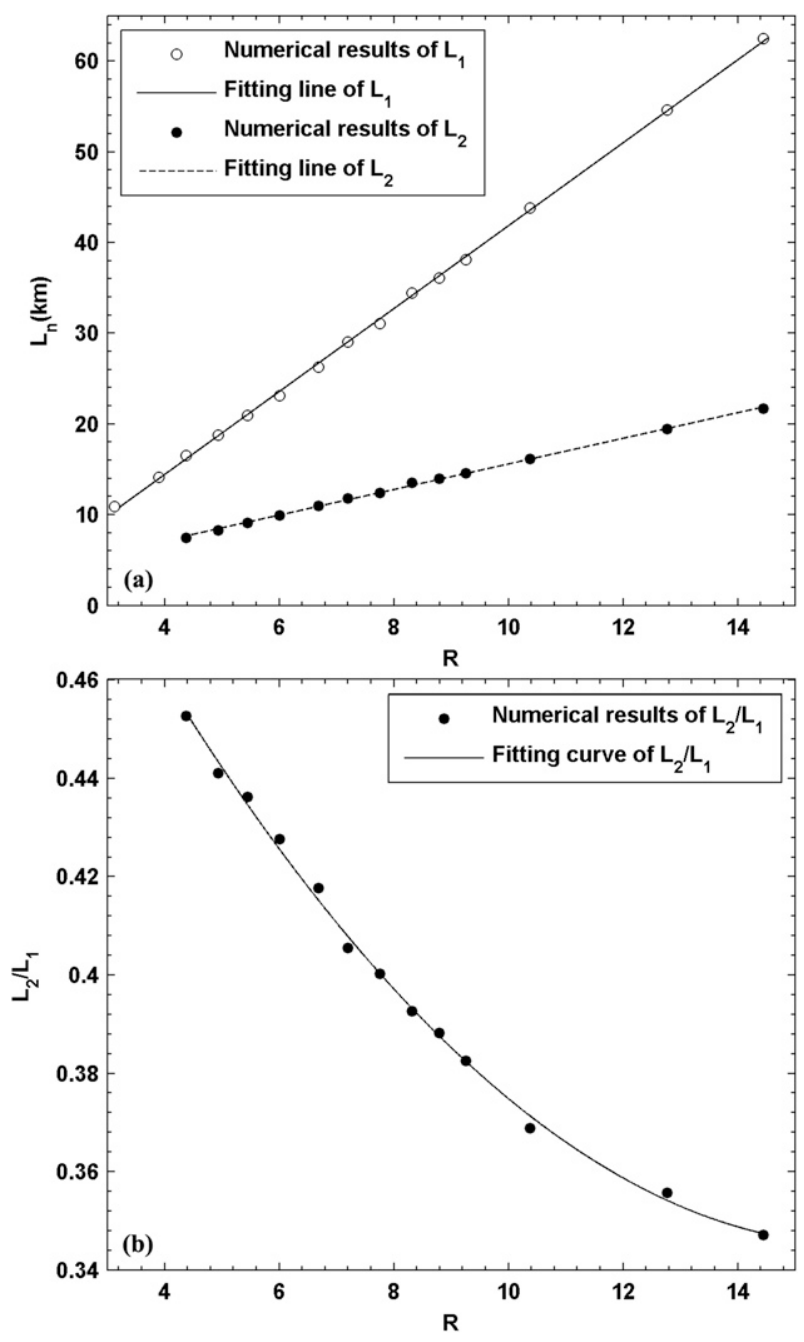

FIG. 9. (a) The distances between two successive wave trains for mode $1\left(L_{1}\right)$ and mode $2\left(L_{2}\right)$ vs $R$ and (b) the ratio of $L_{2} / L_{1}$ vs $R$. The circles and dots show the numerical results. The straight lines and curves are the regressions.

and $E_{2}$ are $E_{1}=\mathrm{KE}_{1}+\mathrm{APE}_{1}$ and $E_{2}=\mathrm{KE}_{2}+\mathrm{APE}_{2}$, respectively. For the mode- 1 internal waves, the highest baroclinic energies appear for $R=\sim 6.0$ in the middle of regime II; however, the nonlinearity of the mode- 1 waves for $R=\sim 6.0$ (Fig. 11b) is not maximal, reflecting the relatively low tidal Froude number $\mathrm{Fr}_{1}^{0}$ (Fig. 3b) and the relatively small percentage contribution to the IWBs from the mode- 1 component (Fig. 7). The nonlinearity $\left(\mathrm{Fr}_{1}\right)$ of the mode- 1 waves for $R=\sim 3.9$ is higher than that for $R=\sim 6.0$ (Fig. 11b), but their baroclinic energies are weaker. This is because the horizontal range $L_{1}$ is proportional to the stratification and the stratification is weaker for $R=\sim 3.9$ (relative to $R=$ 6.0 ), leading to a smaller integral length for the baroclinic energy. For mode- 2 internal waves, the largest baroclinic energies appear for $R=\sim 10.4$ because of the 



FIG. 10. The simulated density fields for the cases N1-N3, N5, N7, N9, N11, N13, and N15 at $t=3 T$ within a nondimensional horizontal distance of $x / L_{1}=3.2$ from the ridge.

stratification effect on the integral length $L_{2}$, while $\mathrm{Fr}_{2}$ is maximal at $R=6.8$. These results show that the baroclinic energies of the radiated internal modes may be high, even when the stratification is not favorable to the generation of strong nonlinearity. Conversely, the stratification suitable for the generation of the most highly nonlinear internal waves does not maximize the baroclinic energy of the generated internal waves. 

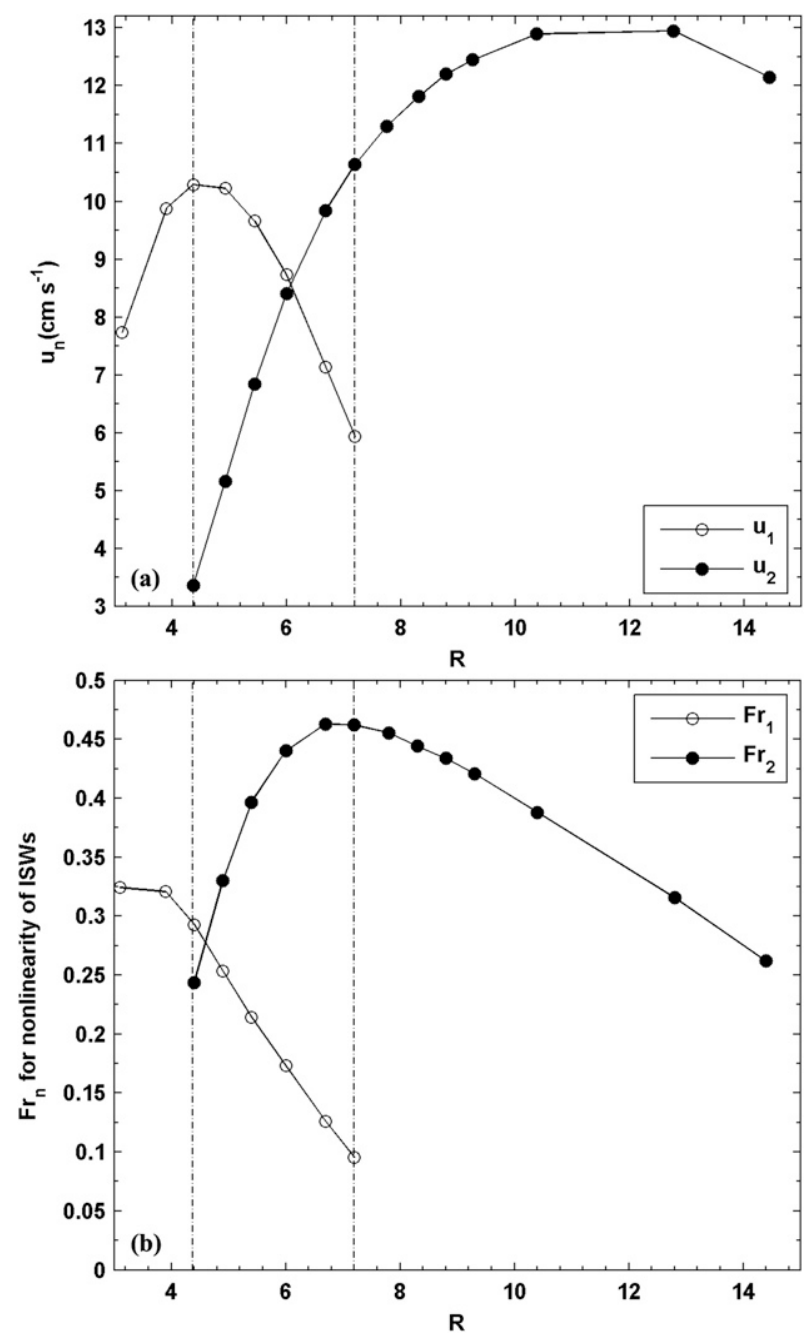

FIG. 11. (a) The wave-induced maximum current $u_{n}$ and (b) the wave Froude number $\mathrm{Fr}_{n}$ for mode-1 (circle) and mode-2 ISWs (dot).

A further question regarding the baroclinic energy is how the partition between $\mathrm{KE}_{n}$ and $\mathrm{APE}_{n}(n=1,2)$ is affected by the stratification. Classical linear and weakly nonlinear theories predict that the energy in a wave train is equally distributed between kinetic and available potential components, but under a fully nonlinear theory (Turkington et al. 1991; Lamb and Nguyen 2009), the kinetic energy is always greater than the available potential energy. Our results show that $\mathrm{KE}_{n} / \mathrm{APE}_{n}>1$ for modes 1 and 2, in agreement with the theory (Fig. 12b). For the mode-1 internal waves, $\mathrm{KE}_{1} / \mathrm{APE}_{1}$ decreases with $R$ because the nonlinearity of the mode- 1 waves (Fig. 11b) decreases with the stratification. In contrast, $\mathrm{KE}_{2} / \mathrm{APE}_{2}$ for the mode- 2 internal waves exhibits a complicated pattern. Its maximum is $<1.1$ in regime II, but it increases rapidly with $R$ in regime III.
This implies that more baroclinic energy of the mode- 2 ISWs remains in the form of $\mathrm{KE}_{2}$ in regime III because the stratification damps the perturbation of the density field and reduces $\mathrm{APE}_{2}$ in the mode-2 ISWs. For shorewardpropagating mode-2 ISWs, Shroyer et al. (2010) showed that the ratio is initially $\sim 1$ and reaches $\sim 3$ after the emergence of short mode-1 linear wave tails. Our results indicate that the $\mathrm{KE}_{2}$ for the mode-2 ISWs is about $25 \%$ higher than the $\mathrm{APE}_{2}$. This result is comparable to $30 \%$ for the mode-1 ISWs estimated by Lamb and Nguyen (2009).

The baroclinic energy radiated in half a tidal period by modes 1 and 2 together (as $E=E_{1}+E_{2}$ ) is shown in Fig. 12c, which reveals that the baroclinic energy is maximal at $R=\sim 6.0$. Thus, based on Figs. 10 and 11a, the baroclinic energy can be radiated out more efficiently if the stratification is favorable for the formation of both internal modes. The ratio of $E_{1}$ to $E_{2}$ (Fig. 12d) shows that the baroclinic energy of the mode- 1 internal waves $\left(E_{1}\right)$ is greater than that of the mode- 2 waves $\left(E_{2}\right)$, implying that the radiated baroclinic energy is mainly carried by the mode- 1 internal waves, not by the mode- 2 internal waves that are prominent in some field observations, even though the mode- 2 waves may be more nonlinear.

Figure 10 shows that the density disturbances of the mode- 1 internal waves in strong stratification cases (e.g., Figs. 10h,i; i.e., for $R \geq \sim 10.4$ ) are hardly discernible, although the total energy of the mode- 1 internal waves is higher than that of the mode- 2 waves. This can be clarified in terms of the spatially averaged energy. The averaged baroclinic kinetic $\overline{\mathrm{KE}}_{n}$ and available potential $\overline{\mathrm{APE}}_{n}$ energies averaged over length scale $L_{n}$ are defined as

$$
\overline{\mathrm{APE}}_{n}=\frac{1}{L_{n}} \int_{x_{n}}^{x_{n}+L_{n}} \int_{H(x)}^{0} E_{a} d z d x,
$$

and

$$
\overline{\mathrm{KE}}_{n}=\frac{1}{L_{n}} \int_{x_{n}}^{x_{n}+L_{n}} \int_{H(x)}^{0} E_{k} d z d x .
$$

The total averaged energy is given by

$$
\bar{E}_{n}=\overline{\mathrm{KE}}_{n}+\overline{\mathrm{APE}}_{n} .
$$

The ratio of $\bar{E}_{1}$ to $\bar{E}_{2}$ is shown in Fig. 13. In contrast to the ratio of $E_{1}$ to $E_{2}, \bar{E}_{1}$ is less than $\bar{E}_{2}$ when $R$ is high (e.g., $R>8.3$ for $W_{0}=2 \mathrm{~km}$ ). Figure 13 shows that the difficulty in discerning the density disturbance of the mode- 1 internal waves in strong stratification cases is due to the increase of the horizontal scale of the mode- 1 

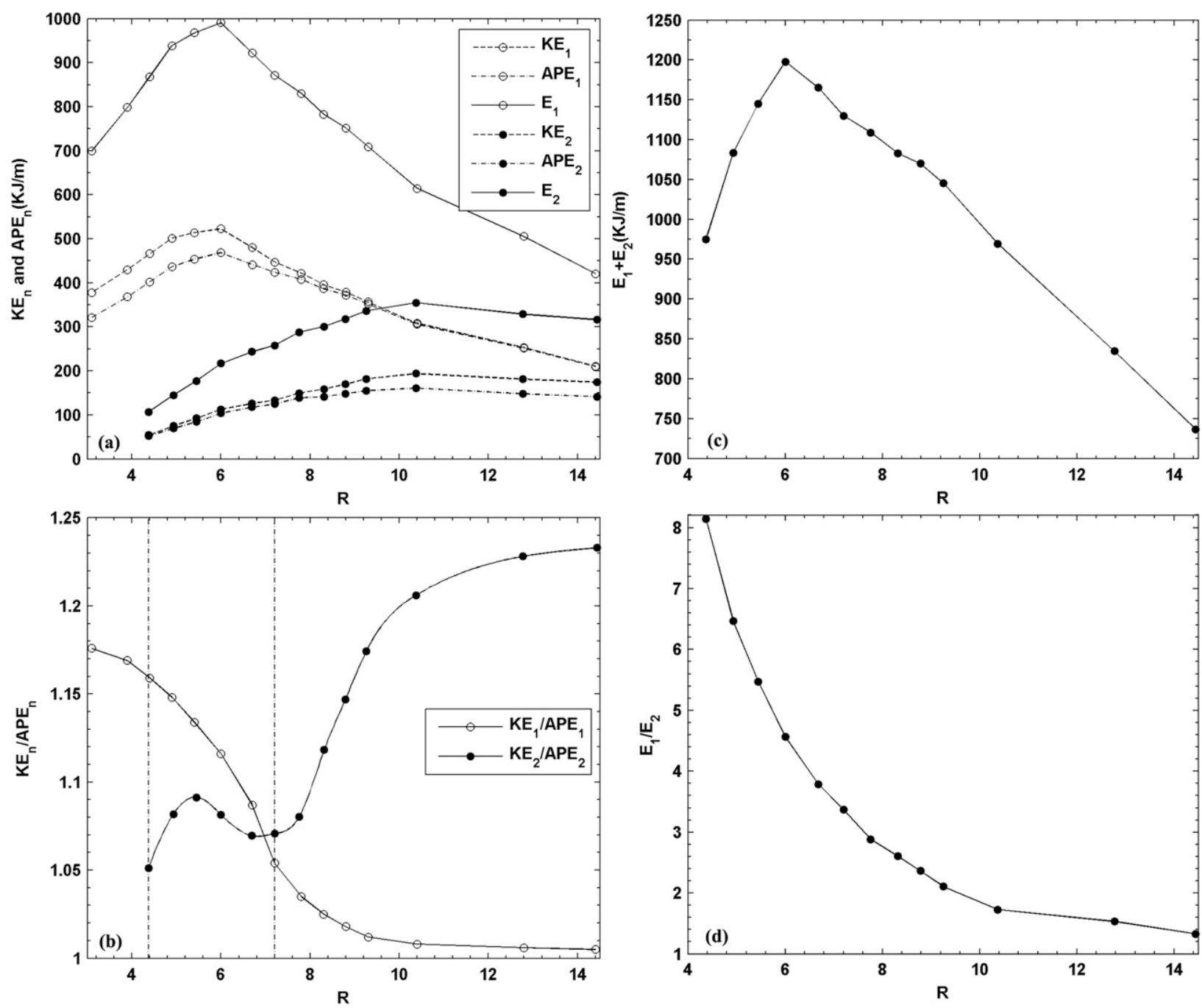

FIG. 12. Baroclinic energy parameters integrated over the wavelength for mode- $n(n=1,2)$ internal wave trains: (a) kinetic energy KE ${ }_{n}$, available potential energy $\mathrm{APE}_{n}$, and the total energy $E_{n}=\mathrm{KE}_{n}+\mathrm{APE}_{n}$; (b) the ratio of kinetic to the available potential energy $\mathrm{KE}_{n} / \mathrm{APE}_{n}$; (c) total energy of the mode- 1 and mode- 2 waves $E_{1}+E_{2}$; and (d) energy ratio $E_{1} / E_{2}$.

waves, which causes the spatially averaged energy to decrease. This also suggests that the spatially averaged baroclinic energy can be used to characterize the density disturbance for different internal modes. On the other hand, the total radiated baroclinic energy calculated from Eqs. (18) and (19) may still be dominated by the mode-1 internal wave, even though it is hardly discernible in the density field in strong stratification cases.

\section{Sensitivity analysis}

The above analyses of stratification effects on tidally induced multimodal internal waves assume that background parameters are held constant. Here, we investigate the sensitivity of internal waves and ISWs to three key background parameters: the tidal forcing, the relative steepness of the ridge, and the depth of the pycnocline.

\section{a. Tidal flux}

The tidal Froude number $\mathrm{Fr}_{n}^{0}$ varies with tidal flux $\psi_{0}$ for all the stratification conditions, and it is helpful to know which wave properties are affected. For all 15 cases N1 to $\mathrm{N} 15$, the tidal flux $\psi_{0}$ was varied from 5 to $35 \mathrm{~m}^{2} \mathrm{~s}^{-1}$. Some wave properties [e.g., the route of IWBs, the percentage contribution to the total variance from each mode, and the characteristic distances $\left(L_{n}\right)$ of the radiated internal waves] are independent of the tidal flux. Accordingly, the numerical results show that they are basically unchanged with different tidal fluxes (results not shown).

The wave nonlinearity parameter $\mathrm{Fr}_{n}$ does, however, vary with the tidal flux $\psi_{0}$. Stratification cases N1 


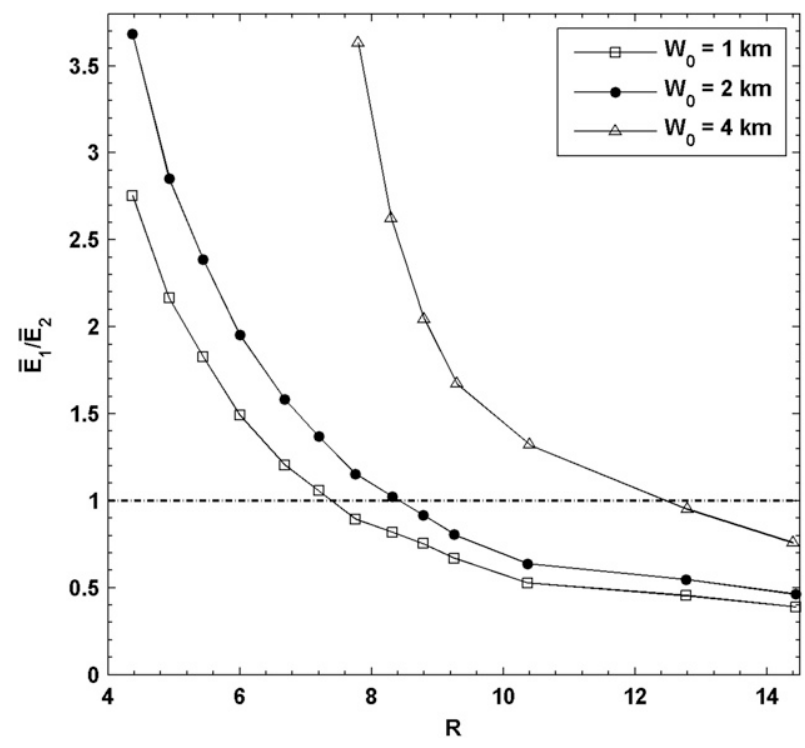

FIG. 13. The ratio of kinetic to available potential energy averaged over the wavelength for three ridge widths: $W_{0}=1 \mathrm{~km}$ (square), $W_{0}=2 \mathrm{~km}$ (circle), and $W_{0}=4 \mathrm{~km}$ (triangle).

(regime I), N8 (regime II), and N15 (regime III) are chosen for analysis. Figures $14 \mathrm{a}$ and $14 \mathrm{~b}$ illustrate, respectively, $\mathrm{Fr}_{1}$ for stratification cases $\mathrm{N} 1$ and $\mathrm{N} 8$ and $\mathrm{Fr}_{2}$ for $\mathrm{N} 8$ and $\mathrm{N} 15$ versus $\psi_{0}$. The nonlinearity of both the mode- 1 and mode- 2 internal waves increases with the tidal flux for all three stratification regimes, but the sensitivity to $\psi_{0}$ depends on the stratification case.
The sensitivity of $\mathrm{KE}_{1} / \mathrm{APE}_{1}$ and $\mathrm{KE}_{2} / \mathrm{APE}_{2}$ to the tidal flux is shown in Figs. $15 \mathrm{a}$ and $15 \mathrm{c}$, respectively. The $\mathrm{KE}_{1} / \mathrm{APE}_{1}$ for case N8 increases with the tidal flux because of the increasing internal wave nonlinearity, while for case $\mathrm{N} 1$ the ratio increases with the tidal flux only up to $\psi_{0}=27.5 \mathrm{~m}^{2} \mathrm{~s}^{-1}$ (Fig. 15a). Figure 15b shows $\mathrm{KE}_{1} / \mathrm{APE}_{1}$ versus $\mathrm{Fr}_{1}$ for the two stratification cases (N1 and N8). The two curves almost coincide, suggesting that the wave nonlinearity is more important than stratification in controlling the partition between KE and APE. For case $\mathrm{N} 1, \mathrm{KE}_{1} / \mathrm{APE}_{1}$ increases with $\mathrm{Fr}_{1}$ initially; however, when the wave nonlinearity parameter $\mathrm{Fr}_{1}$ reaches a critical value at about $\mathrm{Fr}_{1}^{c}=0.35, \mathrm{KE}_{1} / \mathrm{APE}_{1}$ begins to decrease. For case N8, the wave Froude number is always $<\mathrm{Fr}_{1}^{c}$, so $\mathrm{KE}_{1} / \mathrm{APE}_{1}$ increases monotonically with $\mathrm{Fr}_{1}$. For mode- 2 waves, the effect of wave nonlinearity on the energy partition is similar to that for mode- 1 waves, as shown in Figs. 15c and 15d (N8 and N15). The critical Froude number $\mathrm{Fr}_{2}^{c}$ is again $\sim 0.35$. Thus, for both mode- 1 and mode- 2 waves, the ratio of $\mathrm{KE}_{n} / \mathrm{APE}_{n}$ is mainly affected by the wave nonlinearity, which decreases with the wave Froude number if $\mathrm{Fr}_{n}$ is greater than the critical value. In agreement with these simulations, the observations by Scotti et al. (2006) and simulations by Shen et al. (2009) also showed that $\mathrm{KE}_{1} / \mathrm{APE}_{1}$ for shoaling ISWs decreased with the wave nonlinearity above a critical wave Froude number (Staquet and Sommeria 2002).

The ratio of $E_{1}$ to $E_{2}$ is not very sensitive to $\psi_{0}$, and this is consistent with a theoretical prediction (Llewellyn Smith and Young 2002), which suggests that the
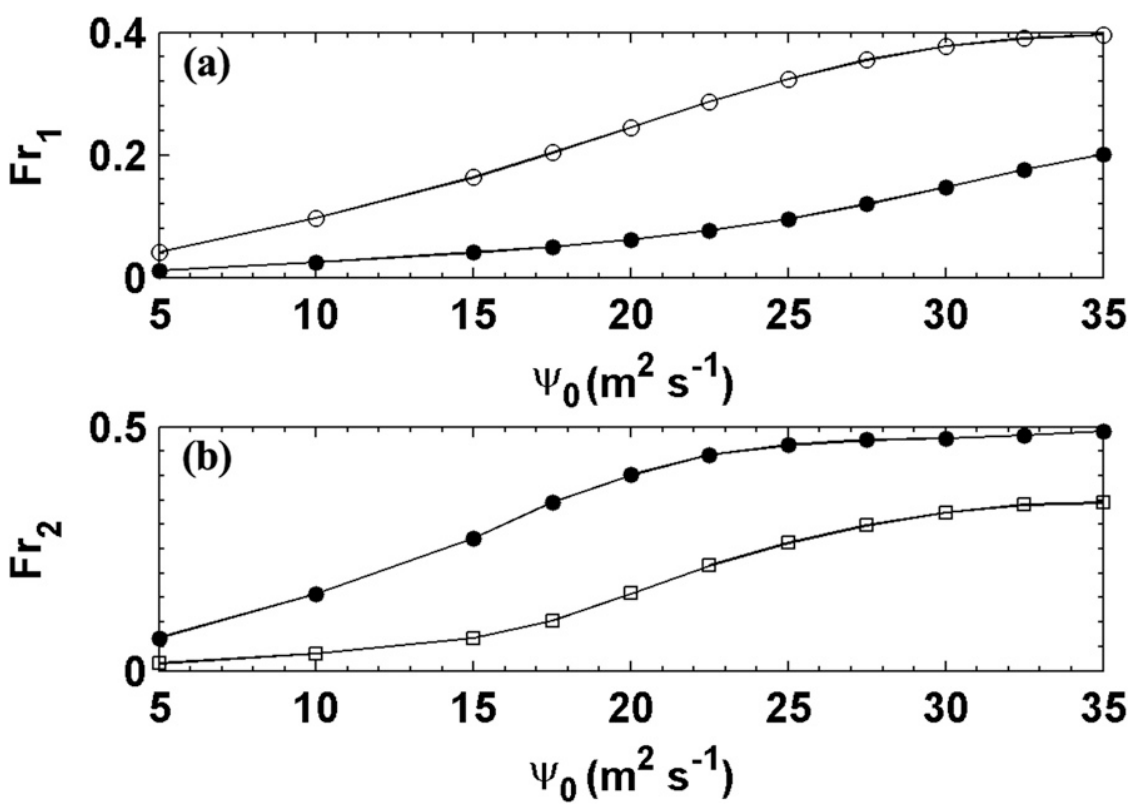

FIG. 14. The $\operatorname{Fr}_{n}(n=1,2)$ vs tidal flux $\psi_{0}$ for stratifications N1 (circle), N8 (solid circle), and $\mathrm{N} 15$ (square). Note that for cases $\mathrm{N} 1$ and N15, only one mode exists. 

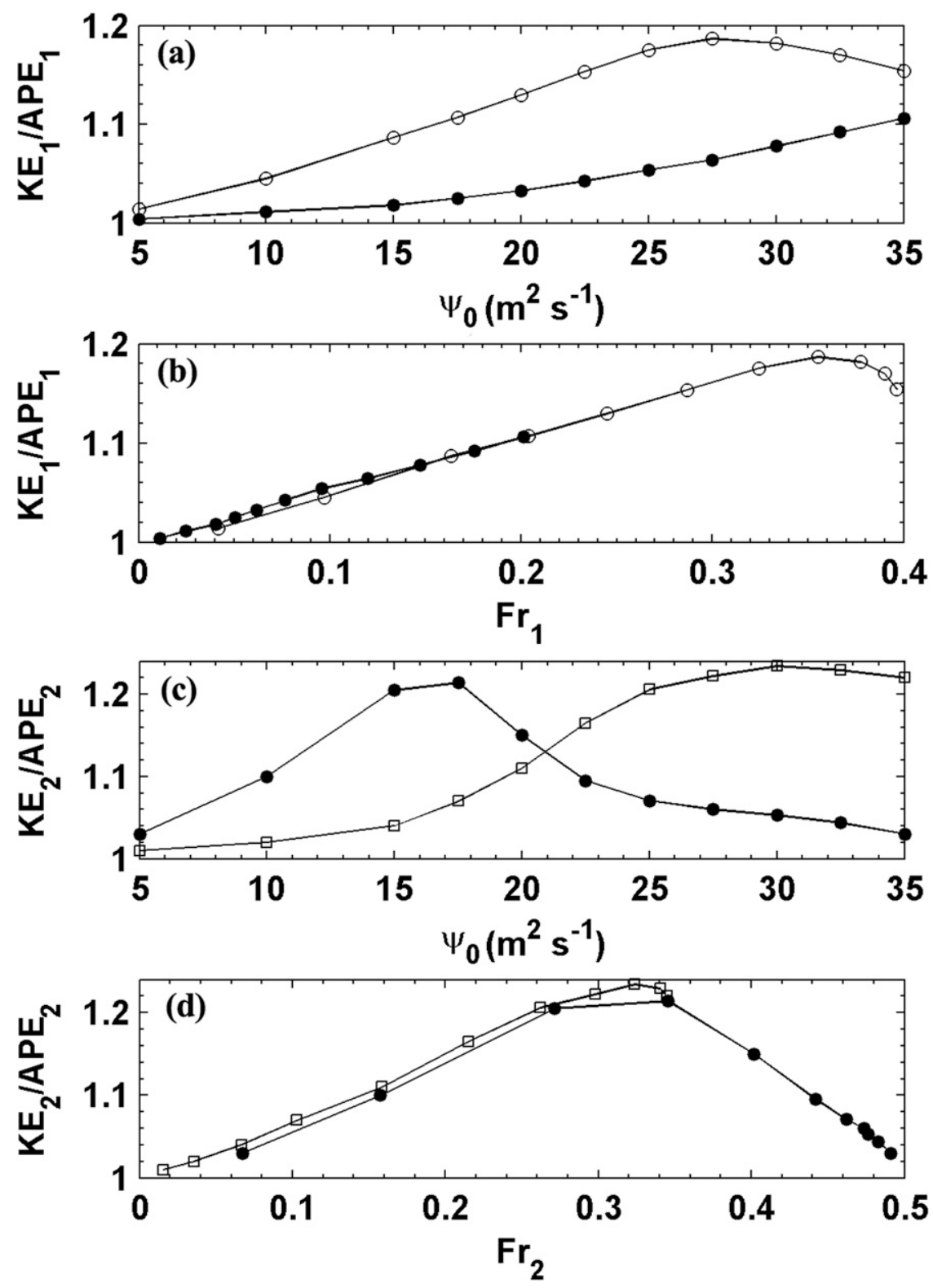

FIG. 15. The ratio of $\mathrm{KE}_{n} / \mathrm{APE}_{n}(n=1,2)$ vs (a),(c) tidal flux $\psi_{0}$ and (b),(d) wave Froude number $\mathrm{Fr}_{n}$ under the stratification N1 (circle), N8 (solid circle), and N15 (square). Note that for cases $\mathrm{N} 1$ and $\mathrm{N} 15$, only one mode exists.

converted energy flux to mode- $n$ internal waves from the barotropic tide is proportional to $U_{0}^{2}$, which is constant. Thus the energy ratio of $E_{1}$ to $E_{2}$ should be independent of the tidal forcing.

\section{b. Ridge relative steepness}

Previous studies have shown that multimodal internal waves can be generated by near-critical and supercritical topography (Vlasenko et al. 2005; Llewellyn Smith and Young 2003). Here, we show that they can also be excited by subcritical ridge topography. To demonstrate the importance of the form of the subcritical topography, two additional subcritical topography sensitivity cases were implemented for all 15 stratification cases. This was carried out by adjusting the width of the ridge $W_{0}$ to 1 and $4 \mathrm{~km}$ instead of $2 \mathrm{~km}$. These widths yield subcritical topography for all 15 stratification cases, with $\gamma=0.443\left(W_{0}=1 \mathrm{~km}\right)$ and $\gamma=0.111\left(W_{0}=4 \mathrm{~km}\right)$; note that $\gamma=0.222$ for $W_{0}=2 \mathrm{~km}$ (case TS1). As $\gamma$ suggests, the effect of stratification on the generation of multimodal internal waves is related to the steepness of the topography and is reflected by the ratio of $\bar{E}_{1} / \bar{E}_{2}$. The 


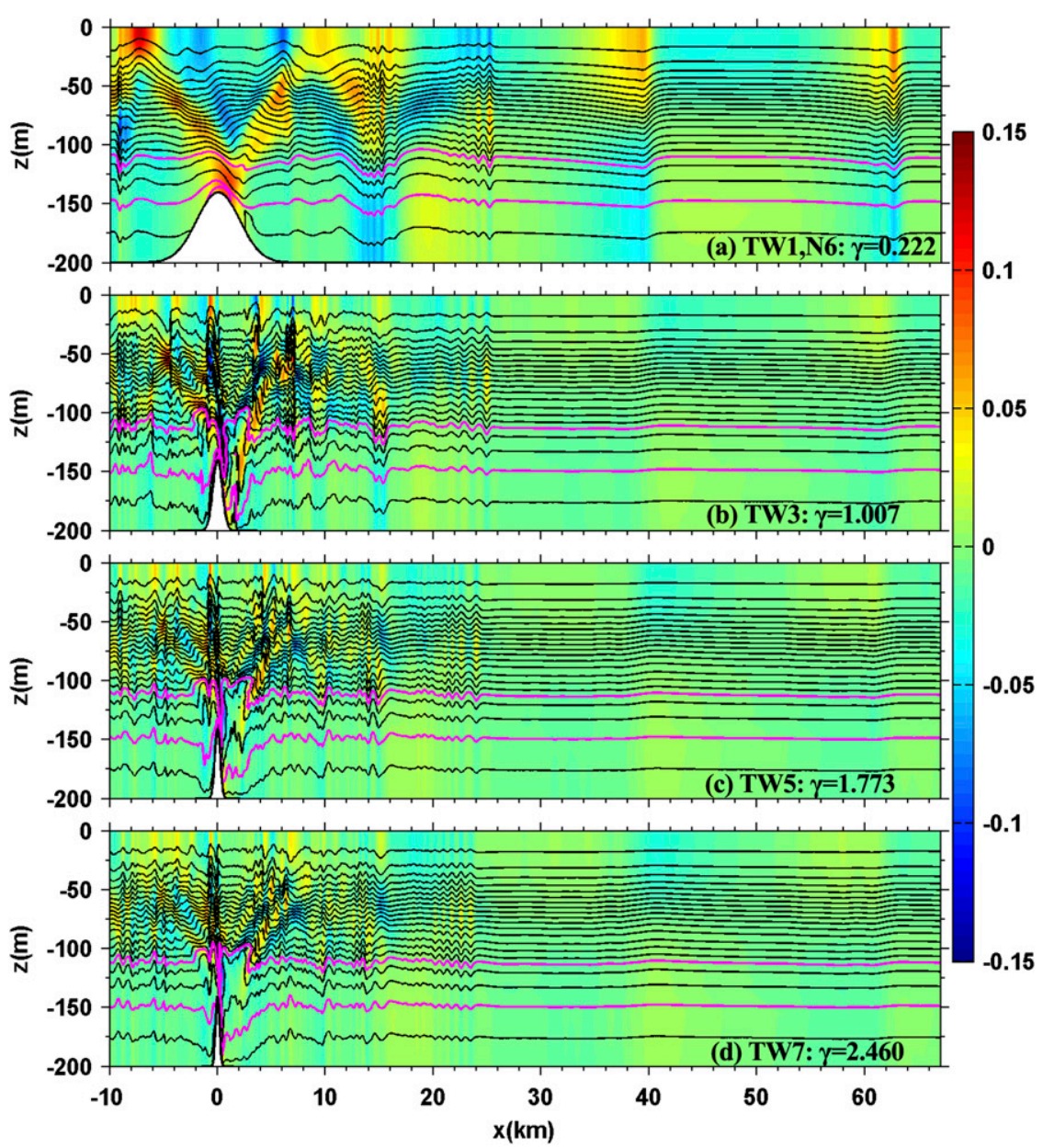

FIG. 16. The simulated density and horizontal velocity fields for the supercritical cases (a) TW1, (b) TW3, (c) TW5, and (d) TW7. The two magenta lines represent the displacement of the isopycnal at depth $z=-110$ and $-150 \mathrm{~m}$.

$\bar{E}_{1} / \bar{E}_{2}$ ratios for $\gamma=0.443\left(W_{0}=1 \mathrm{~km}\right)$ and $\gamma=0.111$ $\left(W_{0}=4 \mathrm{~km}\right)$ are shown in Fig. 13. Figure 13 reveals that mode-2 waves are radiated over steep topography for a wide range of stratification, but over gentle topography (with small $\gamma$ ) the mode- 2 waves can only be radiated for stratification with large values of $R$.

It is also useful to determine what happens when the ridge crest approaches a critical slope and becomes supercritical. With the smaller ridge width $W_{0}$ and the same stratification as in case N6 (i.e., $R=6.0$ ), a sensitivity analysis is performed for near-critical and supercritical slopes, as shown in Table 2 for cases TW1-TW8. Figure 16 shows the velocity and density fields for cases TW1 (Fig. 16a), TW3 (Fig. 16b), TW5 (Fig. 16c), and TW7 (Fig. 16d) and indicates that the beams become narrower with more multimode internal waves generated over the near-critical and supercritical topography than over the subcritical topography. For stratification case N6 (i.e., $R=6.0$ ), the nonlinearity of both mode- 1 and model-2 ISWs decreases with $\gamma$, while the mode-2 wave nonlinearity is higher than that of the model- 1 waves for a wide range of relative ridge steepness (Table 2).

Figure 17 further shows a zoomed-in region over the ridge crest (i.e., $-5 \mathrm{~km} \leq x \leq 5 \mathrm{~km}$ ) for the four cases in Fig. 16. To analyze the results near the ridge crest region, the inner Froude number $\mathrm{Fr}_{t}$ and the inner excursion parameter $\varepsilon$ are used, as defined by Winters and Armi (2013) and Legg and Klymak (2008):

$$
\mathrm{Fr}_{t}=U_{c} /\left(N_{c} h_{0}\right),
$$

and

$$
\varepsilon=\frac{1}{2} \chi^{1 / 2} \frac{\varepsilon_{0}}{\operatorname{Fr}_{0}^{1 / 2}}
$$

respectively, where $N_{c}=2.65 \times 10^{-3} \mathrm{~s}^{-1}$ is the buoyancy frequency at the ridge crest; $\chi=H_{0} /\left(H_{0}-h_{0}\right)$ is 
TABLE 2. Parameters of the supercritical topography sensitivity study cases TW1 to TW8.

\begin{tabular}{|c|c|c|c|c|c|c|c|c|c|}
\hline \multicolumn{2}{|c|}{$\begin{array}{l}\text { Supercritical topography } \\
\text { sensitivity cases }\end{array}$} & \multirow{2}{*}{$\frac{\text { TW1, N6 }}{2.0}$} & \multirow{2}{*}{$\frac{\mathrm{TW} 2}{0.8}$} & \multirow{2}{*}{$\frac{\text { TW3 }}{0.44}$} & \multirow{2}{*}{$\begin{array}{l}\text { TW4 } \\
0.3\end{array}$} & \multirow{2}{*}{$\frac{\text { TW5 }}{0.25}$} & \multirow{2}{*}{$\begin{array}{c}\text { TS6 } \\
0.2\end{array}$} & \multirow{2}{*}{$\frac{\text { TW7 }}{0.18}$} & \multirow{2}{*}{$\frac{\text { TW8 }}{0.12}$} \\
\hline Ridge width $W_{0}(\mathrm{~km})$ & & & & & & & & & \\
\hline Relative steepness $\gamma$ & & 0.222 & 0.554 & 1.007 & 1.476 & 1.773 & 2.206 & 2.460 & 3.690 \\
\hline \multirow[t]{2}{*}{ Wave Froude number } & $\mathrm{Fr}_{1}$ & 0.174 & 0.088 & 0.046 & 0.044 & 0.038 & 0.034 & 0.029 & - \\
\hline & $\mathrm{Fr}_{2}$ & 0.440 & 0.380 & 0.291 & 0.232 & 0.206 & 0.183 & 0.175 & 0.155 \\
\hline \multicolumn{2}{|c|}{ Inner excursion parameter $\varepsilon$} & 0.299 & 0.748 & 1.360 & 1.995 & 2.394 & 2.993 & 3.325 & 4.988 \\
\hline
\end{tabular}

the obstacle gap factor; and $\mathrm{Fr}_{0}=U_{0} /\left(N_{c} h_{0}\right)$ and $\varepsilon_{0}=U_{0} /\left(\sigma W_{0}\right)$ are the outer Froude number and the outer excursion parameter, respectively. Here, $\mathrm{Fr}_{t}$ reaches 1.123 , and the $\varepsilon$ ranges from $\sim 0.3$ to $\sim 5$, as shown in Table 2. The blocking effect is discernible from the isopycnal disturbance at depth $z=\sim-150 \mathrm{~m}$, and the isopycnal is almost completely blocked in Fig. 17d, which suggests that the blocking effect on the upstream denser water is stronger as the ridge becomes narrower, even for a moderate $\mathrm{Fr}_{t}$. Because of the instability and distortion of the upward-propagating IWBs generated over a narrow ridge (Baines 1977; Klymak et al. 2010), the upstream density and velocity fields take on a complex structure, especially for the near-critical cases (e.g., the case TW3 in Fig. 17b).

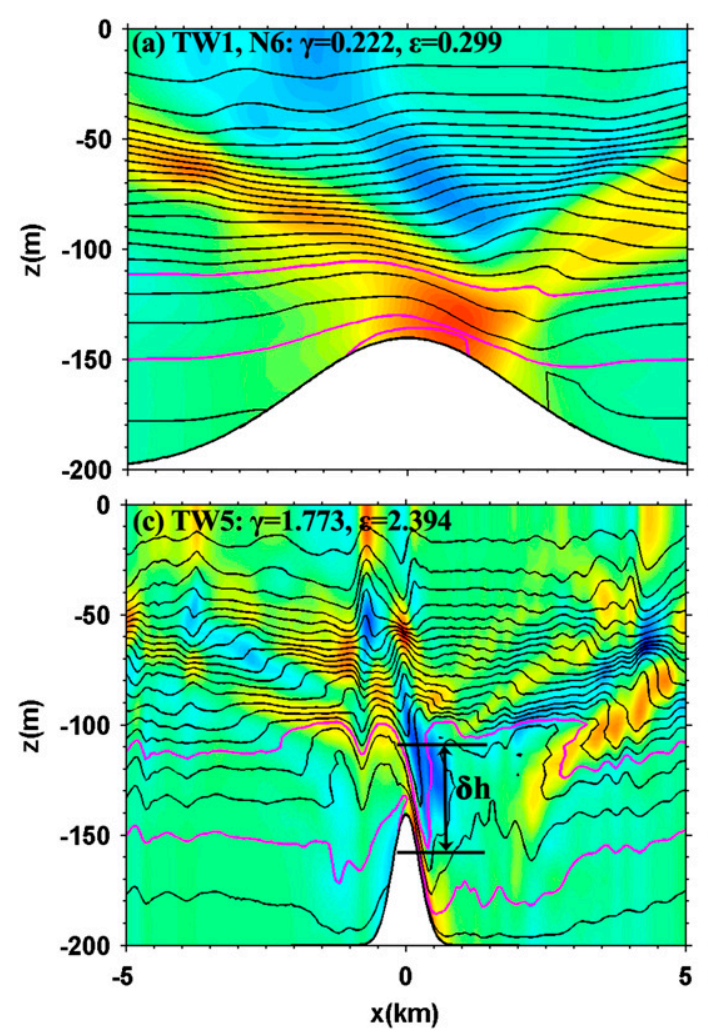

Figure $3 \mathrm{~b}$ emphasizes that smaller values of stratification parameter $R$ lead to larger tidal Froude numbers $\mathrm{Fr}_{n}^{0}$, with possible consequences for lee-wave generation. For instance, if $R<\sim 6.7$, the $\operatorname{Fr}_{2}^{0}$ is $>1$ or $\sim 1$; no obvious internal lee waves are found over subcritical topography (Figs. 10a-e) because the contribution (Fig. 7) from mode- 2 and other higher-mode components to the IWBs are too low. However, for $R=6.0$, Fig. 17 shows that obvious lee waves can be generated over the critical and supercritical topographies because of the high contribution from the higher-mode components to the tidally excited IWBs. This phenomenon, which appears downstream on the lee side of a ridge and is similar to an internal hydraulic jump or the strongly nonlinear quasistationary lee wave (Legg and Klymak 2008), is more

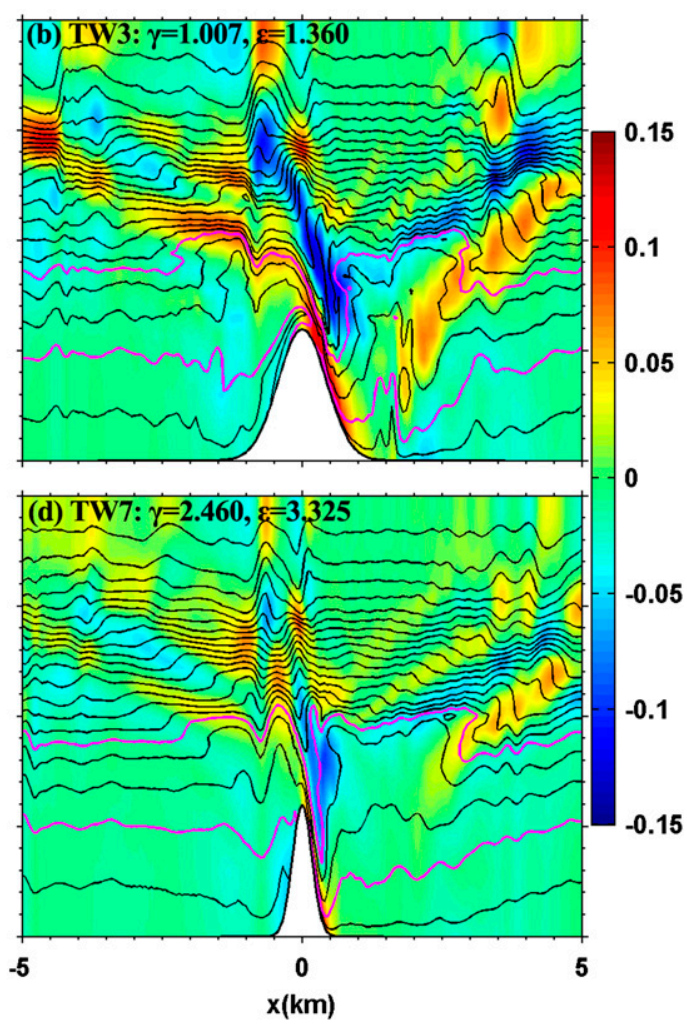

FIG. 17. As in Fig. 16, but in a short region near ridge crest $-5 \mathrm{~km} \leq x \leq 5 \mathrm{~km}$. An example of the $\delta h$ is shown in (c). 


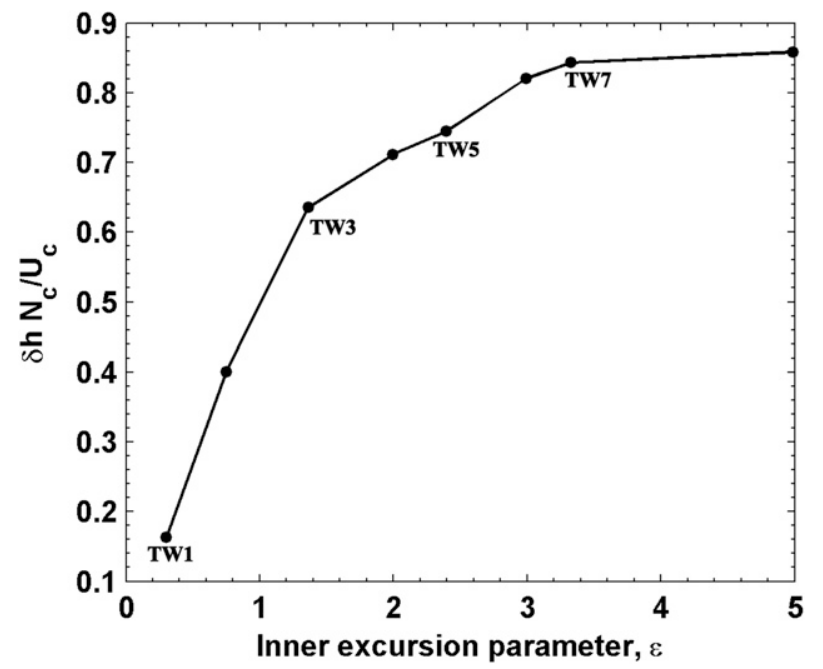

FIG. 18. The nondimensional internal hydraulic jump $\delta h N_{c} / U_{c}$ vs inner excursion parameter.

obvious as the ridge becomes narrower, as shown in Figs. 17b-d. As suggested by Legg and Klymak (2008), we use the maximum displacement of the isopycnal at depth $z=\sim-110 \mathrm{~m}, \delta h$, to scale the magnitude of the internal hydraulic jump (an example of the measured $\delta h$ is shown in Fig. 17c). Here, the isopycnal at depth $z=\sim-110 \mathrm{~m}$ is only partially blocked. Figure 18 shows the nondimensional internal hydraulic jump [defined as the ratio of $\delta h$ to the vertical displacement scale $\left.U_{c} / N_{c}(=67.4 \mathrm{~m})\right]$ versus the inner excursion parameter $\varepsilon$ and reveals that the magnitude of the internal hydraulic jump increases with the inner excursion parameter. A constant $\delta h N_{c} / U_{c}(\approx 0.85)$ is achieved when the inner excursion parameter increases up to $\sim 3$.

\section{c. Pycnocline depth}

It is also useful to explore the sensitivity of internal wave generation to a wide range of pycnocline depth, as shown in cases ND1-ND11 (Fig. 19) by modifying the pycnocline depth $h$ from 5 to $-170 \mathrm{~m}$ based on stratification case N6 (i.e., $R=6.0$ ). Note that case ND1 with $h=5 \mathrm{~m}$ does not have an obvious pycnocline, and the stratification strengthens monotonically toward the surface. The stratification in case ND5 with $h=-65 \mathrm{~m}$ is the same as that in case N6, and at the other extreme, the pycnocline in case ND7 is at middepth with $h=-100 \mathrm{~m}$. The velocity and density fields of six cases (ND1, ND3, ND5, ND7, ND8, and ND10) are shown in Fig. 20, which reveals that high-frequency mode-1 (mode 2) wave trains appear more easily as the pycnocline is located farther away from (closer to) the middepth of the water. In particular, when the stratification does not have an obvious pycnocline such as case ND1

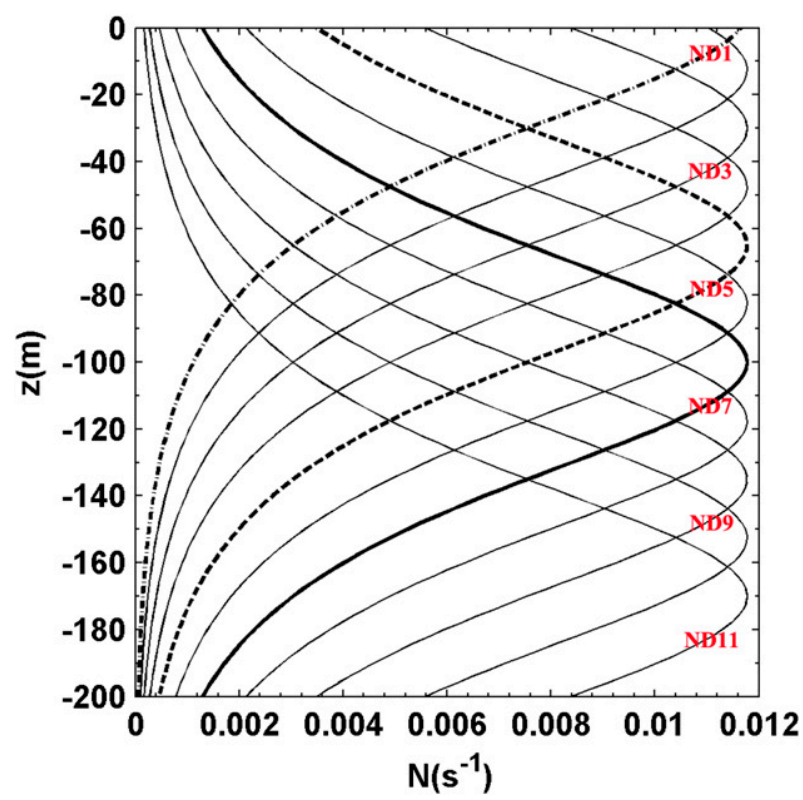

FIG. 19. Stratification profiles ND1 to ND11 for the study of sensitivity to the pycnocline depth: ND1 (thick dotted line), ND5 (thick dashed line), and ND7 (thick line).

(Fig. 20a), high-frequency mode-2 wave trains disappear. The characteristic distances $L_{1}$ and $L_{2}$ of radiated mode- 1 and mode- 2 internal waves versus the pycnocline depth $h$ are illustrated in Fig. 21a, which shows that both $L_{1}$ and $L_{2}$ gradually increase as the pycnocline moves toward middepth. When the pycnocline is above middepth, mode-1 ISWs of depression are excited (Figs. 20a-c); on the contrary, when the pycnocline is below middepth, mode-1 ISWs of elevation are generated (Figs. 20e,f). As mentioned in section 4a, the wave nonlinearity is a determinant factor in controlling the partition between KE and APE; thus, it is of great significance to clarify how the wave nonlinearity parameter $\mathrm{Fr}_{n}$ of the radiated mode- 1 and mode- 2 internal waves vary with the pycnocline depth $h$. As revealed in Fig. 21b, the nonlinearity of the excited mode- 1 internal waves tends to increase if the pycnocline moves toward the sea surface or the water bottom, and the nonlinearity of the excited mode- 2 internal waves increases if the pycnocline approaches the middepth.

Further, when the pycnocline deepens below the ridge top, the buoyancy frequency at the ridge crest $N_{c}$ increases and the inner Froude number $\mathrm{Fr}_{t}$ decreases. Thus, the blocking effect near the ridge crest region in Fig. 20 is much more significant. Meanwhile, because of the decreasing outer Froude number $\mathrm{Fr}_{0}$ for the deep pycnocline, the inner excursion parameter $\varepsilon$ increases, so that downstream, as shown in Fig. 20, the internal hydraulic jumps become stronger as the pycnocline 


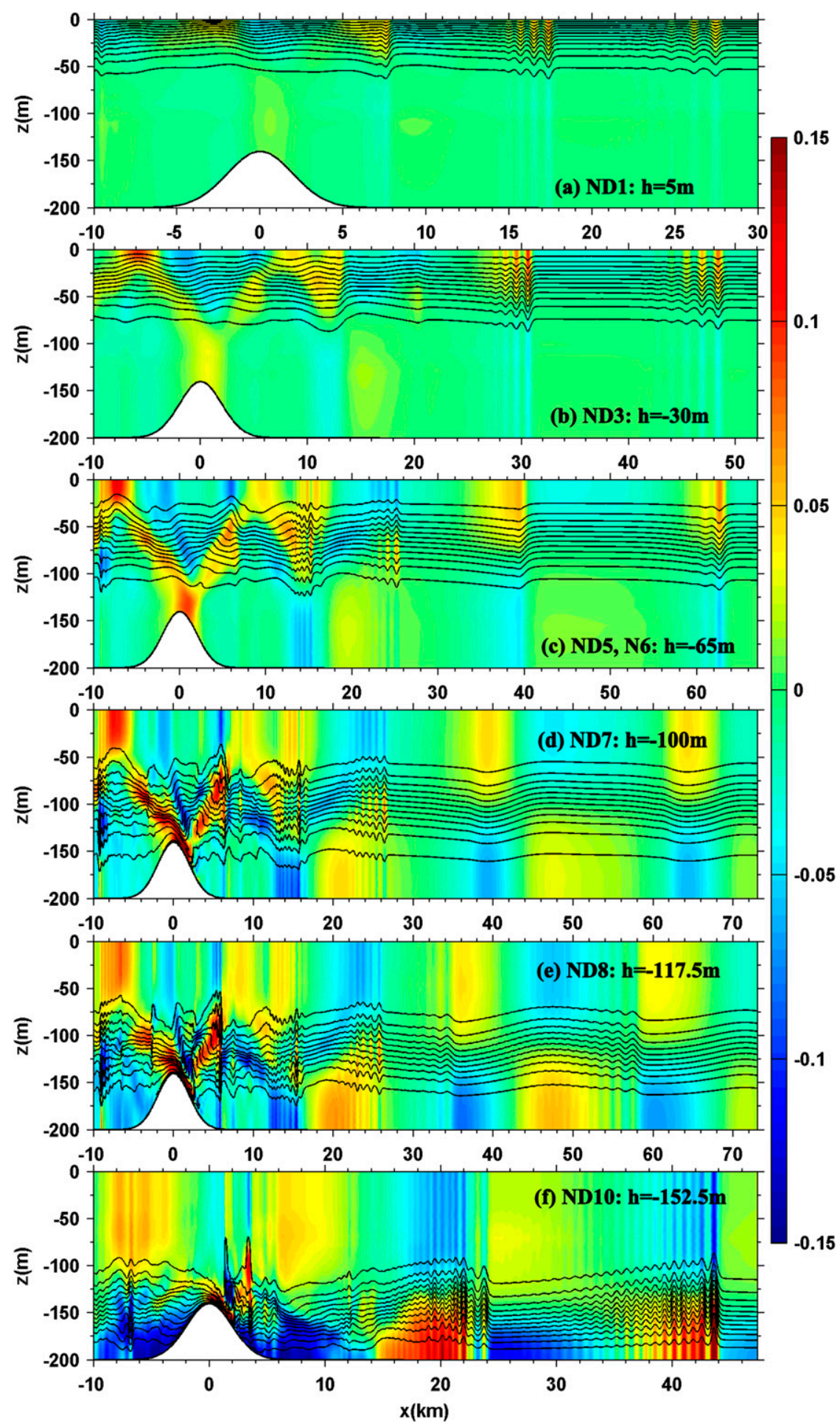

FIG. 20. The simulated density and horizontal velocity fields for the sensitivity cases (a) ND1, (b) ND3, (c) ND5, (d) ND7, (e) ND8, and (f) ND10. (Note the difference in the horizontal scales due to the different horizontal wavelengths for stratifications with different pycnocline depth $h$.) 

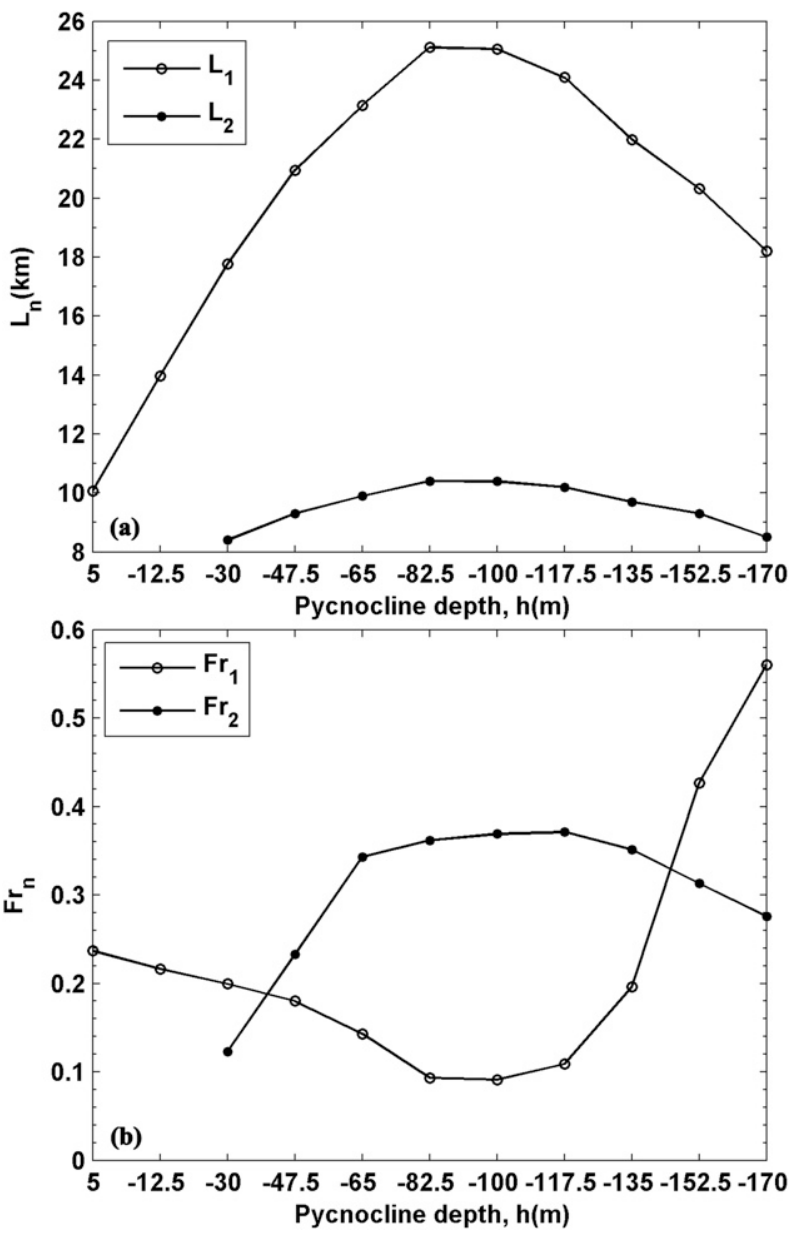

FIG. 21. (a) The distances between two successive wave trains $L_{n}$ and (b) the wave Froude number $\mathrm{Fr}_{n}$ vs pycnocline depth $h$ for mode-1 (circle) and mode-2 ISWs (dot).

moves toward the water bottom and is below the ridge top.

\section{Discussion}

The topography of the Luzon Strait (LS) in the South China Sea (SCS) displays the double-ridge characteristics, which includes the east Lan-Yu Ridge (LR) and the west Heng-Chun Ridge (HR) (Cai et al. 2012; Buijsman et al. 2012). The topography of sections A $\left(20^{\circ} 05^{\prime} \mathrm{N}\right), \mathrm{B}$ $\left(21^{\circ} 20^{\prime} \mathrm{N}\right)$, and $\mathrm{C}\left(20^{\circ} 30^{\prime} \mathrm{N}\right)$ (Fig. 22a) across the LS with six ridges is shown in Fig. 22b. The summer stratification (Fig. 22c) near the LS from Levitus (2009) is chosen to define the relative steepness of the six ridges (Table 3 ). In section $\mathrm{C}$, the relative steepness $\gamma$ of the west (lowest) ridge $\mathrm{C}_{\mathrm{HR}}$ is $<0.1$, and therefore the topography in this section can be regarded as having a single ridge $C_{L R}$. For ridges $A_{H R}$ and $B_{L R}$, results of this study suggest that both ridges may play an important role in generation of multimodal internal waves when the strength of the upper-ocean stratification is strong, even though the relative steepness is $<1$.

Remote sensing and in situ observations show that mode- 1 and mode- 2 ISWs exist in the continental shelf region of the northern SCS and vary with upper-ocean stratification (Zheng et al. 2007; Yang et al. 2009). It is found that summer, when pycnocline depth is shallow, is the most favorable season for the generation of mode-1 ISWs, while the winter season, with a deep pycnocline depth, is most favorable for generation of mode-2 ISWs. This seasonal variation of the mode- 1 and mode- 2 ISWs resulting from the pycnocline depth change in the continental shelf region of the northern SCS is consistent with the sensitivity analysis result in section 4c. However, strong mode-2 ISWs have also been observed near the source site in the LS during summer, during periods when upper-ocean stratification is strong and shallow (Ramp et al. 2012). These observations imply that mode-2 internal waves can appear during periods of strong stratification, which validates the results of this modeling study that the moderate and strong upperocean stratification is also favorable for the generation of the mode-2 ISWs.

\section{Conclusions}

A 2.5-dimensional nonhydrostatic model with an adjustable vertical resolution was developed to investigate the effects of the stratification in the upper ocean on multimodal internal waves generated by tidal flow over a subcritical ridge, where only upward-propagating IWBs are present. Propagation routes of the simulated IWBs are in good agreement with calculated characteristic lines.

One important result of this study is that stratification effects on internal waves can be described in terms of three upper-ocean stratification regimes as weak, moderate, and strong. Under weak stratification $(R \leq \sim 4.0)$, weak IWBs with $\leq 2$ modes are excited, and the mode- 1 component is dominant. For moderate stratification $(\sim 4.0 \leq R \leq \sim 7.5)$, IWBs of moderate strength and consisting of 2-4 modes can be generated; the percentage contribution from the mode- 2 component is comparable to the mode- 1 waves. In strong stratification ( $R \geq \sim 7.5$ ), strong IWBs of five or more modes can be generated; however, the tidal Froude number is too small to support evolution of the mode-1 ISWs, so that only the mode- 1 baroclinic tide can be excited. In a moderately stratified upper ocean, both mode- 1 and mode-2 ISWs can be produced efficiently.

The distances between the two successive wave trains, $L_{1}$ and $L_{2}$ (respectively) for mode- 1 and mode- 2 internal 

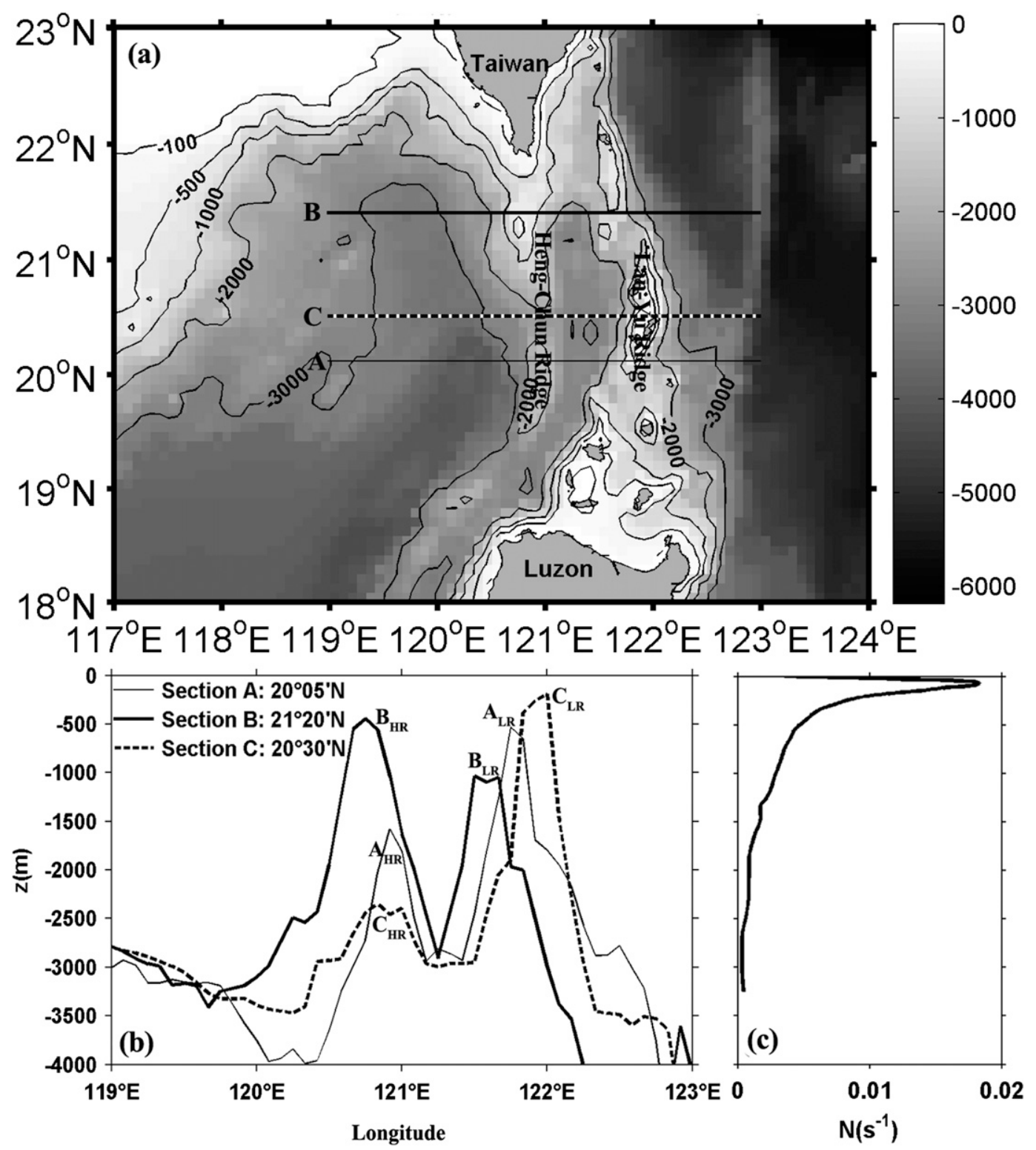

FIG. 22. (a) Sections A, B, and C across the Luzon Strait along $20^{\circ} 05^{\prime} \mathrm{N}, 21^{\circ} 20^{\prime} \mathrm{N}$, and $20^{\circ} 30^{\prime} \mathrm{N}$, (b) the topography along sections A, B, and C, and (c) the summer stratification near the Luzon Strait from Levitus (2009) data.

waves, increase linearly with the stratification above the ridge. Model results predict that the baroclinic energies of the radiated mode- $n(n=1,2)$ internal wave $E_{n}$ are high for certain values of the stratification parameter $R$, specifically for $R=\sim 6.0$ for the mode- 1 waves and for $R=\sim 10.4$ for mode- 2 waves. The radiated baroclinic energy may still be dominated by the mode- 1 internal wave, although the mode- 2 internal waves are obvious visually, while the mode- 1 waves are hardly discernable.

An important contribution of this study is to define ISW mode structure relative to upper-ocean stratification and other parameters. The spatial structure of the
IWBs generated at a subcritical ridge is not so sensitive to the tidal flux $\psi_{0}$, whereas the internal wave nonlinearity and energy partition are both affected by $\psi_{0}$. As the topography becomes steeper (i.e., as $\gamma$ increases), IWBs display more multimodal characteristics, and mode- 2 waves are radiated over steep topography for a wide range of stratification, but are only radiated over gentle topography (small $\gamma$ ) for strong stratification with large $R$. Topography-generated multimodal internal waves are sensitive to not only the pycnocline depth but also the upper-ocean stratification strength. Subcritical ridges also exist in the LS of the northern SCS. This 
TABLE 3. Parameters of the six ridges of sections A $\left(20^{\circ} 05^{\prime} \mathrm{N}\right), \mathrm{B}$ $\left(21^{\circ} 20^{\prime} \mathrm{N}\right)$, and $\mathrm{C}\left(20^{\circ} 30^{\prime} \mathrm{N}\right)$ across the LS.

\begin{tabular}{ccc}
\hline \hline Ridges in the LS & $h_{0}(\mathrm{~m})$ & Relative steepness $\gamma$ \\
\hline $\mathrm{A}_{\mathrm{LR}}$ & 2464 & 1.4 \\
$\mathrm{~A}_{\mathrm{HR}}$ & 1418 & 0.3 \\
$\mathrm{~B}_{\mathrm{LR}}$ & 1964 & 0.5 \\
$\mathrm{~B}_{\mathrm{HR}}$ & 2556 & 1.1 \\
$\mathrm{C}_{\mathrm{LR}}$ & 2800 & 2.6 \\
$\mathrm{C}_{\mathrm{HR}}$ & 653 & $<0.1$ \\
\hline
\end{tabular}

study implies that they may play an important role in the generation of multimodal internal waves in the LS during summer when the strength of the upper-ocean stratification is strong.

Acknowledgments. This work is supported by the General Research Fund of Hong Kong Research Grants Council (RGC) under Grants CUHK 402912 and 403113 (Xie and Pan), the Hong Kong Innovation and Technology Fund under the Grants ITS/321/13 and ITS/ 259/12 (Pan), the direct grants of the Chinese University of Hong Kong (Pan), the National Natural Science Foundation of China under Projects 41406025, 41430964, and 41025019 (Xie) and 41376035 (Pan), the NSFC Grant OCE-0851527 (Dynamics of a Rotating, Supercritical Large-River Plume, Pan and Jay), and OCE-0929055 (Secular Changes in Pacific Tides, Jay). The authors are grateful to the anonymous reviewers for their valuable and important comments and suggestions.

\section{REFERENCES}

Akylas, T. R., and R. H. J. Grimshaw, 1992: Solitary internal waves with oscillatory tails. J. Fluid Mech., 242, 279-298, doi:10.1017/ S0022112092002374.

,,-- S. R. Clarke, and A. Tabaei, 2007: Reflecting tidal wave beams and local generation of solitary waves in the ocean thermocline. J. Fluid Mech., 593, 297-313, doi:10.1017/ S0022112007008786.

Baines, P. G., 1977: Upstream influence and Long's model in stratified flows. J. Fluid Mech., 82, 147-159, doi:10.1017/ S0022112077000573.

Balmforth, N. J., G. R. Ierley, and W. R. Young, 2002: Tidal conversion by subcritical topography. J. Phys. Oceanogr., 32, 2900-2914, doi:10.1175/1520-0485(2002)032<2900: TCBST $>2.0 . \mathrm{CO} ; 2$.

Bogucki, D., L. G. Redekopp, and J. Barth, 2005: Internal solitary waves in the Coastal Mixing and Optics 1996 experiment: Modal structure and resuspension. J. Geophys. Res., 110, C02024, doi:10.1029/2003JC002253.

Buijsman, M. C., Y. Kanarska, and J. C. McWilliams, 2010: On the generation and evolution of nonlinear internal waves in the South China Sea. J. Geophys. Res., 115, C02012, doi:10.1029/ 2009JC005275.

— S. Legg, and J. Klymak, 2012: Double-ridge internal tide interference and its effect on dissipation in Luzon Strait. J. Phys. Oceanogr., 42, 1337-1356, doi:10.1175/JPO-D-11-0210.1.
Cai, S., J. Xie, and J. He, 2012: An overview of internal solitary waves in the South China Sea. Surv. Geophys., 33, 927-943, doi:10.1007/s10712-012-9176-0.

Colosi, J. A., and W. Munk, 2006: Tales of the venerable Honolulu tide gauge. J. Phys. Oceanogr., 36, 967-996, doi:10.1175/ JPO2876.1.

Farmer, D. M., and J. D. Smith, 1980: Tidal interaction of stratified flow with a sill in Knight Inlet. Deep-Sea Res., 27A, 239-254, doi:10.1016/0198-0149(80)90015-1.

Gerkema, T., 2001: Internal and interfacial tides: Beam scattering and local generation of solitary waves. J. Mar. Res., 59, 227255, doi:10.1357/002224001762882646.

, and J. T. F. Zimmerman, 2008: An introduction to internal waves. Royal NIOZ Lecture Notes, 207 pp. [Available online at www.nioz.nl/files/upload/users/258010/book.pdf.]

Grisouard, N., C. Staquet, and T. Gerkema, 2011: Generation of internal solitary waves in a pycnocline by an internal wave beam: A numerical study. J. Fluid Mech., 676, 491-513, doi:10.1017/jfm.2011.61.

Hall, R. A., J. M. Huthnance, and R. G. Williams, 2013: Internal wave reflection on shelf slopes with depth-varying stratification.J. Phys. Oceanogr., 43, 248-258, doi:10.1175/ JPO-D-11-0192.1.

Helfrich, K. R., and W. K. Melville, 1986: On long nonlinear internal waves over slope-shelf topography. J. Fluid Mech., 167, 285-308, doi:10.1017/S0022112086002823.

Jackson, C. R., 2004: An atlas of internal solitary-like waves and their properties. 2nd ed. Global Ocean Associates Rep., 560 pp. [Available online at www.internalwaveatlas.com/ Atlas2_index.html.]

Kang, D., 2010: Energetics and dynamics of internal tides in Monterey Bay using numerical simulations. Ph.D. dissertation, Stanford University, $170 \mathrm{pp}$

Kantha, L. H., and C. A. Clayson, 2000: Numerical Models of Oceans and Oceanic Processes. Academic Press, $940 \mathrm{pp}$.

Klymak, J. M., S. Legg, and R. Pinkel, 2010: A simple parameterization of turbulent tidal mixing near supercritical topography. J. Phys. Oceanogr., 40, 2059-2074, doi:10.1175/ 2010JPO4396.1.

Lamb, K. G., 2004: Nonlinear interaction among internal wave beams generated by tidal flow over supercritical topography. Geophys. Res. Lett., 31, L09313, doi:10.1029/2003GL019393.

- 2008: On the calculation of the available potential energy of an isolated perturbation in a density stratified fluid. J. Fluid Mech., 597, 415-427, doi:10.1017/S0022112007009743.

— solitary waves with an application to reflectance. J. Phys. Oceanogr., 39, 559-580, doi:10.1175/2008JPO3882.1.

Legg, S., and J. Klymak, 2008: Internal hydraulic jumps and overturning generated by tidal flow over a tall steep ridge. J. Phys. Oceanogr., 38, 1949-1964, doi:10.1175/2008JPO3777.1.

Levitus, S., Ed., 2009: World Ocean Atlas 2009. U.S. Government Printing Office, 1110 pp. [Available online at www.nodc.noaa. gov/OC5/WOA09/pr_woa09.html.]

Liu, A. K., F. C. Su, M. K. Hsu, N. J. Kuo, and C. R. Ho, 2013: Generation and evolution of mode-two internal waves in the South China Sea. Cont. Shelf Res., 59, 18-27, doi:10.1016/ j.csr.2013.02.009.

Llewellyn Smith, S. G., and W. R. Young, 2002: Conversion of the barotropic tide. J. Phys. Oceanogr., 32, 1554-1566, doi:10.1175/1520-0485(2002)032<1554:COTBT >2.0.CO;2.

, and _ 2003: Tidal conversion at a very steep ridge.J. Fluid Mech., 495, 175-191, doi:10.1017/S0022112003006098. 
Mercier, M. J., M. Mathur, L. Gostiaux, T. Gerkema, J. M. Magalhães, J. C. B. da Silva, and T. Dauxois, 2012: Soliton generation by internal tidal beams impinging on a pycnocline: Laboratory experiments. J. Fluid Mech., 704, 37-60, doi:10.1017/jfm.2012.191.

Nash, J. D., E. Kunze, C. M. Lee, and T. B. Sanford, 2006: Structure of the baroclinic tide generated at Kaena Ridge, Hawaii. J. Phys. Oceanogr., 36, 1123-1135, doi:10.1175/JPO2883.1.

New, A. L., and R. D. Pingree, 1990: Large-amplitude internal soliton packets in the central Bay of Biscay. Deep-Sea Res., 37, 513-524, doi:10.1016/0198-0149(90)90022-N.

North, G. R., L. B. Thomas, F. C. Robert, and J. M. Fanthune, 1982: Sampling errors in the estimation of empirical orthogonal functions. Mon. Wea. Rev., 110, 699-706, doi:10.1175/ 1520-0493(1982)110<0699:SEITEO > 2.0.CO;2.

Osborne, A. R., and T. L. Burch, 1980: Internal solitons in the Andaman Sea. Science, 208, 451-460, doi:10.1126/ science.208.4443.451.

Pickering, A., and M. H. Alford, 2012: Velocity structure of internal tide beams emanating from Kaena Ridge, Hawaii. J. Phys. Oceanogr., 42, 1039-1044, doi:10.1175/JPO-D-12-018.1.

Ramp, S. R., Y. J. Yang, D. B. Reeder, and F. L. Bahr, 2012: Observations of a mode-2 nonlinear internal wave on the northern Heng-Chun Ridge south of Taiwan. J. Geophys. Res., 117, C03043, doi:10.1029/2011JC007662.

Scotti, A., R. Beardsley, and B. Butman, 2006: On the interpretation of energy and energy fluxes of nonlinear internal waves: An example from Massachusetts Bay. J. Fluid Mech., 561, 103-112, doi:10.1017/S0022112006000991.

Shen, C. Y., T. E. Evans, R. M. Oba, and S. Finette, 2009: Three-dimensional hindcast simulation of internal soliton propagation in the Asian Seas International Acoustics Experiment area. J. Geophys. Res., 114, C01014, doi:10.1029/ 2008JC004937.

Shroyer, E. L., J. N. Moum, and J. D. Nash, 2010: Mode 2 waves on the continental shelf: Ephemeral components of the nonlinear internal wavefield. J. Geophys. Res., 115, C07001, doi:10.1029/ 2009JC005605.

Staquet, C., and J. Sommeria, 2002: Internal gravity waves: From instabilities to turbulence. Annu. Rev. Fluid Dyn., 34, 559-593, doi:10.1146/annurev.fluid.34.090601.130953.

Stastna, M., and W. Peltier, 2005: On the resonant generation of large amplitude internal solitary waves and solitary-like waves. J. Fluid Mech., 543, 267-292, doi:10.1017/ S002211200500652X.

Turkington, B., A. Eydeland, and S. Wang, 1991: A computational method for solitary internal waves in a continuously stratified fluid. Stud. Appl. Math., 85, 93-127.

Vazquez, A., N. Stashchuk, V. Vlasenko, M. Bruno, A. Izquierdo, and P. C. Gallacher, 2006: Evidence of multimodal structure of the baroclinic tide in the Strait of Gibraltar. Geophys. Res. Lett., 33, L17605, doi:10.1029/2006GL026806.

Venayagamoorthy, S. K., and O. B. Fringer, 2007: On the formation and propagation of nonlinear internal boluses across a shelf break. J. Fluid Mech., 577, 137-159, doi:10.1017/ S0022112007004624.

Vitousek, S., and O. B. Fringer, 2011: Physical vs. numerical dispersion in nonhydrostatic ocean modeling. Ocean Modell., 40, 72-86, doi:10.1016/j.ocemod.2011.07.002.

Vlasenko, V., and W. Alpers, 2005: Generation of secondary internal waves by the interaction of an internal solitary wave with an underwater bank. J. Geophys. Res., 110, C02019, doi:10.1029/2004JC002467.

— - N. Stashchuk, and K. Hutter, 2005: Baroclinic Tides: Theoretical Modeling and Observational Evidence. Cambridge University Press, $351 \mathrm{pp}$.

, C. Guo, and X. Chen, 2010: Multimodal structure of baroclinic tides in the South China Sea. Nonlinear Processes Geophys., 17, 529-543, doi:10.5194/npg-17-529-2010.

Winters, B. K., and L. Armi, 2013: The response of a continuously stratified fluid to an oscillating flow past an obstacle. J. Fluid Mech., 727, 83-118, doi:10.1017/jfm.2013.247.

Xie, J., S. Cai, and Y. He, 2010: A continuously stratified nonlinear model for internal solitary waves in the northern South China Sea. Chin. J. Oceanol. Limnol., 28, 1040-1048, doi:10.1007/ s00343-010-9077-3.

Yang, Y. J., Y. C. Feng, M.-H. Chang, S. R. Ramp, C.-C. Kao, and T.-Y. Tang, 2009: Observations of second baroclinic mode internal solitary waves on the continental slope of the northern South China Sea. J. Geophys. Res., 114, C10003, doi:10.1029/ 2009JC005318.

Zheng, Q., R. D. Susanto, C.-R. Ho, Y. Song, and Q. Xu, 2007: Statistical and dynamical analyses of generation mechanisms of solitary internal waves in the northern South China Sea. J. Geophys. Res., 112, C03021, doi:10.1029/2006JC003551. 
Copyright of Journal of Physical Oceanography is the property of American Meteorological Society and its content may not be copied or emailed to multiple sites or posted to a listserv without the copyright holder's express written permission. However, users may print, download, or email articles for individual use. 